\title{
Validation of Galileo orbits using SLR with a focus on satellites launched into incorrect orbital planes
}

\author{
Krzysztof Sośnica $^{1}\left(\mathbb{D} \cdot\right.$ Lars Prange $^{2} \cdot$ Kamil Kaźmierski $^{1} \cdot$ Grzegorz Bury $^{1}$. \\ Mateusz Drożdżewski $^{1}$ - Radosław Zajdel ${ }^{1}$ - Tomasz Hadas ${ }^{1}$
}

Received: 20 October 2016 / Accepted: 8 July 2017 / Published online: 24 July 2017

(C) The Author(s) 2017. This article is an open access publication

\begin{abstract}
The space segment of the European Global Navigation Satellite System (GNSS) Galileo consists of In-Orbit Validation (IOV) and Full Operational Capability (FOC) spacecraft. The first pair of FOC satellites was launched into an incorrect, highly eccentric orbital plane with a lower than nominal inclination angle. All Galileo satellites are equipped with satellite laser ranging (SLR) retroreflectors which allow, for example, for the assessment of the orbit quality or for the SLR-GNSS co-location in space. The number of SLR observations to Galileo satellites has been continuously increasing thanks to a series of intensive campaigns devoted to SLR tracking of GNSS satellites initiated by the International Laser Ranging Service. This paper assesses systematic effects and quality of Galileo orbits using SLR data with a main focus on Galileo satellites launched into incorrect orbits. We compare the SLR observations with respect to microwave-based Galileo orbits generated by the Center for Orbit Determination in Europe (CODE) in the framework of the International GNSS Service Multi-GNSS Experiment for the period 2014.0-2016.5. We analyze the SLR signature effect, which is characterized by the dependency of SLR residuals with respect to various incidence angles of laser beams for stations equipped with single-photon and multiphoton detectors. Surprisingly, the CODE orbit quality of satellites in the incorrect orbital planes is not worse than that of nominal FOC and IOV orbits. The RMS of SLR residuals is even lower by 5.0 and $1.5 \mathrm{~mm}$ for satellites in the incorrect
\end{abstract}

Krzysztof Sośnica

krzysztof.sosnica@igig.up.wroc.pl

1 Institute of Geodesy and Geoinformatics, Wrocław University of Environmental and Life Sciences, Grunwaldzka 53, Wrocław, Poland

2 Astronomical Institute, University of Bern, Sidlerstrasse 5, Bern, Switzerland orbital planes than for FOC and IOV satellites, respectively. The mean SLR offsets equal $-44.9,-35.0$, and $-22.4 \mathrm{~mm}$ for IOV, FOC, and satellites in the incorrect orbital plane. Finally, we found that the empirical orbit models, which were originally designed for precise orbit determination of GNSS satellites in circular orbits, provide fully appropriate results also for highly eccentric orbits with variable linear and angular velocities.

Keywords GNSS - Galileo - Precise orbit determination · ECOM $\cdot$ Orbit modeling $\cdot$ Satellite laser ranging $\cdot$ Satellite signature effect

\section{Introduction}

Galileo is the European Global Navigation Satellite System (GNSS) that is currently at the deployment stage. Two early test satellites, called Galileo In-Orbit Validation Element-A (GIOVE-A) and GIOVE-B, were launched in 2005 and in 2008 , respectively. These test satellites were used to validate critical technology components, as well as to secure the Galileo frequencies (Montenbruck et al. 2015a). GIOVE-A and GIOVE-B were decommissioned in mid-2012 after the successful launch of their successors, i.e., the first pair of full-featured In-Orbit Validation (IOV) Galileo spacecraft (Steigenberger and Montenbruck 2017). In total, four IOV spacecraft were launched between 2011 and 2012.

In 2014, the first pair of Full Operational Capability (FOC) Galileo satellites was launched on a Soyuz ST rocket, however, into wrong, highly eccentric orbits. Instead of a nominal altitude of 23,225 km above the Earth's surface with an inclination of $56^{\circ}$ and a revolution period of $14 \mathrm{~h} 05 \mathrm{~m}$, the satellites were orbiting at heights between 13.713 and $25,900 \mathrm{~km}$ with an inclination of $50.1^{\circ}$ and a revolution period of $11 \mathrm{~h} 42 \mathrm{~m}$. A 

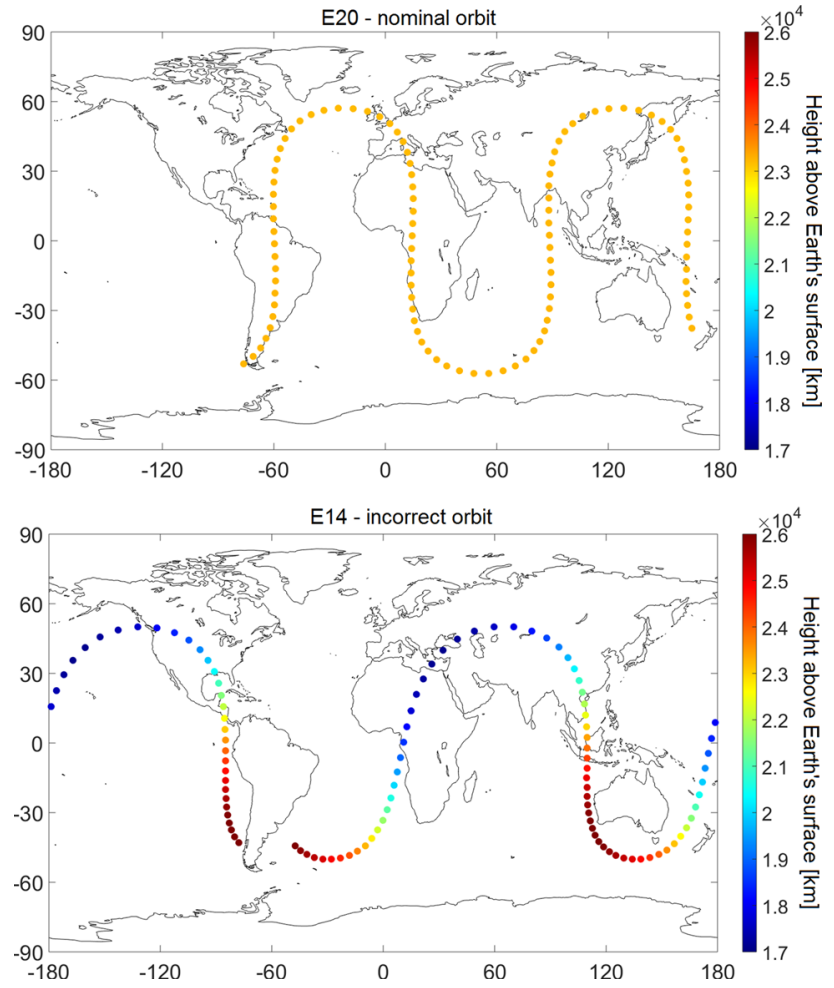

Fig. 1 Groundtracks of Galileo satellite positions for the arc length of $26 \mathrm{~h}$ with a 15 -min sampling interval for the nominal orbit (top) and incorrect orbit (bottom). Color bar corresponds to satellite height above the Earth's surface. A clear difference between satellite's velocity in perigee (dark blue) and apogee (red) can be observed for E14 (dots are plotted every $15 \mathrm{~min}$ )

series of satellite maneuvers allowed for a correction of the eccentricity at the cost of substantial fuel consumption. Currently the satellites orbit with a revolution period of $12 \mathrm{~h} 56 \mathrm{~m}$ at heights between 17,178 and 26,019 km (see Fig. 1), which reduces the radiation exposure from the Van Allen radiation belts. Although the perigee was substantially increased, the orbits are still highly eccentric with $e=0.1585$, whereas the orbital inclination angle could not be corrected at all. These two FOC satellites cannot be included in the almanac of the broadcast navigation message, because the eccentricity and the semi-major axis exceed the limit of the deviation w.r.t. the nominal orbit, which amounts to $\Delta e_{\max }=0.03125$ and $\Delta a_{\max }=87 \mathrm{~km}$ (Steigenberger and Montenbruck 2017).

In 2015 another six and in May 2016 another two FOC Galileo satellites were launched, all of which reached their target orbits. As a result, today there are 14 active Galileo satellites: 12 in three nominal orbital planes: A, B, and C; and 2 in the fourth 'abnormal' or 'extended' plane.

\subsection{SLR tracking of Galileo spacecraft}

As opposed to the current constellation of GPS satellites, all Galileo spacecraft are nominally equipped with laser retroreflector arrays (LRA) designed for satellite laser ranging (SLR). Galileo LRAs consist of 84 and 60 corner cube retroreflectors for IOV and FOC, respectively. The fused silica corner cubes are uncoated on the rear reflecting side for both IOV and FOC satellites, whereas the front side of IOV cubes is coated by the anti-reflection indium tin oxide ${ }^{1}$ for $532 \mathrm{~nm}$ (Dell'Agnello 2010). The uncoated corner cubes are preferable, as they increase the return rate signal strength so that the target is easier to acquire by the laser stations (Wilkinson and Appleby 2011). The test GIOVE-A and GIOVE-B satellites were equipped with aluminum-coated retroreflectors; thus, their return rates were typically lower than currently for the IOV and FOC satellites.

Tracking high-orbiting GNSS satellites poses a challenge for laser stations: on the one hand due to a weak returning signal in the presence of background noise especially during the daytime and on the other hand due to an increasing number of targets scheduled for SLR tracking. However, SLR stations with a proper automation and scheduling can track more than 140 different satellites (Kirchner and Koidl 2015).

The International Laser Ranging Service (ILRS) (Pearlman et al. 2002) initiated three GNSS intensive tracking campaigns between 2014 and 2015 in order to improve the scheduling, to increase the data volume especially in the daytime, and to find the best possible tracking strategy which is a trade-off between the maximum number of registered satellite pass segments and the maximum number of tracked satellites (ILRS 2014). The GNSS tracking campaigns were coordinated by the ILRS Study Group LARGE (LAser Ranging to GNSS s/c Experiment) which was appointed tasked in 2014 to promote SLR tracking of GNSS satellites.

The SLR observations to GNSS satellites allows validating the GNSS microwave orbits (Pavlis 1995; Urschl et al. 2007; Montenbruck et al. 2015b), combining SLR and GNSS techniques for the improvement of GNSS orbits' quality (Hackel et al. 2015) or using co-locations in space for the estimation of the satellite microwave antenna offsets, LRA offsets, and the scale transfer from the SLR to GNSS solutions (Thaller et al. 2011, 2014, 2015).

\subsection{IGS MGEX campaign}

In 2012, the International GNSS Service (IGS) (Dow et al. 2009) established the Multi-GNSS experiment (MGEX) (Montenbruck et al. 2013) in order to prepare operational services providing precise orbit and clock products for new and upcoming GNSS, such as the European Galileo, Chinese BeiDou, and regional systems, such as Japanese QuasiZenith Satellite System (QZSS) and Indian NAVigation with Indian Constellation (NAVIC) system. The multi-GNSS solutions are a challenge for MGEX analysis centers due

\footnotetext{
${ }^{1}$ http://ilrs.gsfc.nasa.gov/docs/ILRS_MSR_Galileo_201106.pdf.
} 
to the increasing number of satellites, new signals and observations types, and orbit modeling difficulties caused by different attitude steering modes as well as different satellites' sensitivities to perturbing forces. Currently seven MGEX Analysis Centers provide orbit and clock products of at least one new GNSS: (1) Center for Orbit Determination in Europe (CODE), Switzerland (Prange et al. 2016), (2) Centre National d'Études Spatiales (CNES), France (Loyer et al. 2012), (3) Deutsches GeoForschungsZentrum (GFZ), Germany (Uhlemann et al. 2016), (4) Technische Universität München (TUM), Germany (Steigenberger et al. 2013), (5) Wuhan University (WU), China (Guo et al. 2015), (6) Japan Aero-space Exploration Agency (JAXA), Japan (Kasho 2014), and (7) European Space Agency (ESA), Europe (Springer et al. 2012). Comparisons of orbit and clocks provided by different MGEX Analysis Centers can be found in Steigenberger et al. (2015); Guo et al. (2017), and Prange et al. (2017).

\subsection{The objective and the structure of this paper}

This paper evaluates systematic effects and the quality of Galileo orbits using SLR data with a main focus on Galileo satellites launched into incorrect orbits. Section 2 provides general information on the SLR and GNSS data collecting and processing strategies. Section 3 describes the current space segment of the Galileo system with differences between satellites and the orbit characteristics. Sections 4 and 6 provide an analysis of SLR residuals to individual Galileo satellites, different orbital planes, different types of Galileo spacecraft, and different empirical orbit models. Section 5 discusses the issues related to multi-photon and single-photon SLR detector types. The results are summarized and concluded in the last section.

\section{GNSS and SLR solutions}

\subsection{CODE MGEX solutions}

CODE, as one of the MGEX analysis centers, was providing three system solutions based on GPS, GLONASS, Galileo in 2012. BeiDou (MEO and IGSO) was additionally included in late 2013. Since 2014 CODE has been providing five satellite system solutions based on GPS, GLONASS, Galileo, BeiDou, and QZSS (Prange et al. 2017) on an operational basis. CODE orbit solutions are based on double-difference microwave GNSS phase observations using the ionospherefree linear combination. For Galileo, the linear combination is formed on a basis of E1 and E5a signals. The orbits are always referred to the middle day of 3-day arcs, because the 3-day solutions are typically associated with much smaller misclosures at the orbit boundaries, as well as better esti-

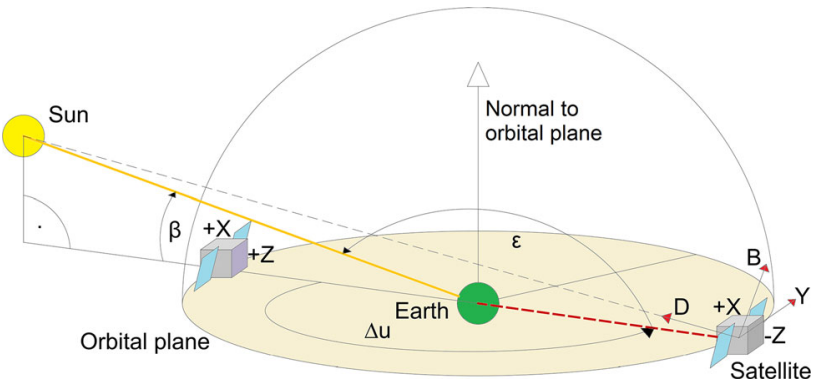

Fig. 2 Sun-Earth-satellite reference frame showing the elevation angle of the Sun over orbital plane $\beta$, argument of satellite latitude with respect to the Sun $\Delta u$ and the elongation angle $\varepsilon$

mates of Earth rotation parameters when new or incomplete GNSS systems are involved (Lutz et al. 2016). The observation sampling is $180 \mathrm{~s}$ with the elevation cutoff angle equal to $3^{\circ}$.

In this paper, we analyze the SLR observation residuals with respect to microwave Galileo orbits generated by CODE for the period between January 2014 and July 2016. In January 2015, CODE changed the orbit model from the classical Empirical CODE Orbit Model (ECOM1) (Beutler et al. 1994; Springer et al. 1999) to the extended ECOM2 (Arnold et al. 2015). In order to keep a full consistency throughout the entire analyzed time series, for 2015 and 2016 the operational CODE MGEX solutions are used, whereas for 2014 the reprocessed orbits using ECOM2 are employed, instead of operational MGEX products based on ECOM1.

The orbit definition consists of 6 Keplerian parameters for the initial epoch of a satellite arc and up to 9 empirical orbit parameters, decomposed into three orthogonal directions: the $D$ axis pointing from the satellite toward the Sun, the $Y$ axis lying along the solar panels, and the $B$ axis completing the right-handed coordinate orthogonal frame (see Fig. 2 for an explanation).

The classical ECOM1 has been used for generating highprecision GPS orbits by most of the IGS Analysis Centers. ECOM1 includes the estimation of 5 empirical parameters: $D_{0}, Y_{0}, B_{0}, B_{C}$, and $B_{S}$ (Beutler et al. 1994). Recent studies revealed, however, that for new GNSS satellites, such as GLONASS-M (Arnold et al. 2015) or Galileo (Montenbruck et al. 2015b; Prange et al. 2017) the classical ECOM1 introduces spurious signals into orbit and clock products. Therefore, the new model, i.e., ECOM2, was developed for a better absorption of the solar radiation pressure acting upon the elongated satellite bodies (Arnold et al. 2015). ECOM 2 was originally derived for GLONASS-M as it remarkably reduces spurious periodic signals in the combined GPS + GLONASS products.

When using ECOM2, a set of 9 empirical parameters is estimated: $D_{0}, Y_{0}, B_{0}, D_{C 2}, D_{S 2}, D_{C 4}, D_{S 4}, B_{C}$, and $B_{S}$ (Arnold et al. 2015). All parameters are estimated without 
any constraints. No a priori solar radiation pressure model, no albedo model, and no antenna thrust are used for Galileo orbits generated at CODE. The penultimate section of this paper (Sect. 6) provides the comparison between Galileo orbits based on ECOM1 and ECOM2.

It is assumed that all Galileo satellites are subject to the yaw steering attitude control strategy, despite that for very low $|\beta|$ angles of less than $2^{\circ}$ a patented dynamic yaw steering is in fact employed (Ebert and Oesterlin 2008). In the dynamic yaw steering mode, the yaw angle is replaced by a smoothed version to keep the maximum slew rate at noon and midnight within the defined threshold, which also avoids discontinuities in the yaw rate and its higher-order derivatives (Montenbruck et al. 2015a). Neglect of the dynamic yaw steering modeling may lead to some orbit errors, but only for very low $|\beta|$ angles.

Besides the satellite orbits, CODE solutions include the following parameters: GNSS station coordinates, $2 \mathrm{~h}$ tropospheric zenith path delays, $24 \mathrm{~h}$ tropospheric horizontal gradients, and $24 \mathrm{~h}$ Earth rotation parameters ( $X$ and $Y$ pole coordinates, their drifts, and the excess of length of day). The no-net-rotation minimum constraint is imposed on a subset of stable GNSS stations. The same software, i.e., the Bernese GNSS Software 5.3 (Dach et al. 2015) is used along with the same a priori models for microwave as well as for SLR solutions. For more details on CODE MGEX solutions, see Prange et al. (2017).

\subsection{SLR solutions}

The SLR observation residuals are computed as differences between laser ranges and the microwave-based positions of GNSS satellites. SLR provides the accuracy assessment mainly for the radial component, because the maximum nadir angle of SLR observations for the Galileo satellites in nominal orbits is $12.3^{\circ}$, whereas for the Galileo satellites in highly eccentric orbits the maximum nadir angle varies between $11.3^{\circ}$ and $15.5^{\circ}$ in apogee and perigee, respectively.

The SLR station coordinates are fixed to the a priori reference frame SLRF2008. ${ }^{2}$ GNSS-derived Earth rotation parameters from CODE MGEX solutions are used for the transformation between the Earth-fixed and inertial reference frames. The LRA offset values provided by ESA have been applied in order to refer the SLR observations to the spacecraft's center-of-mass. ${ }^{3}$ The station displacement models, including solid Earth tides, ocean tidal loading, and the mean pole definition are consistent with the International Earth Rotation and Reference System Service (IERS) Con-

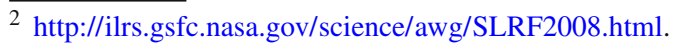

${ }^{3}$ http://ilrs.gsfc.nasa.gov/missions/satellite_missions/current_missi ons/ga01_com.html.
}

ventions 2010 (Petit and Luzum 2011) and thus also with the microwave-based GNSS solutions.

We employ a two-stage residual screening for SLR data. In the first step, only the largest residuals exceeding the $1-\mathrm{m}$ level are excluded. Then, the mean values and standard deviations (sigma) are calculated. Finally, those SLR residuals which exceed the threshold of mean \pm 3 sigma are excluded and new mean and sigma values are calculated again. More details on SLR validation of GNSS orbits at CODE can be found in Sośnica et al. (2015).

One has to keep in mind that the SLR observations, despite their high precision, may not be fully free of biases (Appleby et al. 2016). Therefore, the ILRS Analysis Standing Committee established a pilot project in order to investigate the SLR range biases over the long time spans for LAGEOS and Etalon satellites. Initial results indicate differences between biases to mid-orbiting LAGEOS and high-orbiting Etalons of the order of $20-30 \mathrm{~mm}$ for SLR stations equipped with the multi-photon detectors and virtually no biases for SLR stations equipped with single-photon detectors (Appleby and Rodríguez 2016). Similar differences in SLR offsets between multi-photon and single-photon stations were also found when analyzing GLONASS observations (Sośnica et al. 2015).

Figure 3 shows statistics concerning the SLR observations collected to different Galileo satellites as a function of time and laser station. An outstanding performance, in terms of number of collected data, can be observed for Yarragadee (7090), Mt. Stromlo (7825), Changchun (7237), Graz (7839), Wettzell (8834), Herstmonceux (7840), Zimmerwald (7810), and Monument Peak (7110). The largest number of data was collected to the IOV satellites: E11, E12, E19, especially between August and October 2015, i.e., during the third ILRS GNSS intensive tracking campaign.

\section{Galileo orbits}

The Galileo satellite constellation is planned to be a Walker constellation with 27 satellites in circular orbits distributed over 3 orbital planes separated by $120^{\circ}$ with an additional inactive spare satellite in each plane, all of which orbit at the inclination angle $i=56^{\circ}$ (see Table 1). The satellites' altitude of about 23,225 km above the Earth's surface corresponds to a semi-major axis of about $29,600 \mathrm{~km}$ and revolution period of $14 \mathrm{~h} 05 \mathrm{~m}$. Every satellite completes 17 orbital revolutions in 10 sidereal days. The draconitic year of Galileo satellites, i.e., a period of two consecutive passes of the Sun through the orbital plane in the same direction, equals 355.6 days, which is by about 3 days longer than that for GPS satellites. The maximum $\beta$ angle for Galileo is planeand time-dependent, but never exceeds $80^{\circ}$ (see Table 1). The area of the IOV solar panels and of the $X$ and $Z$ sides of the 

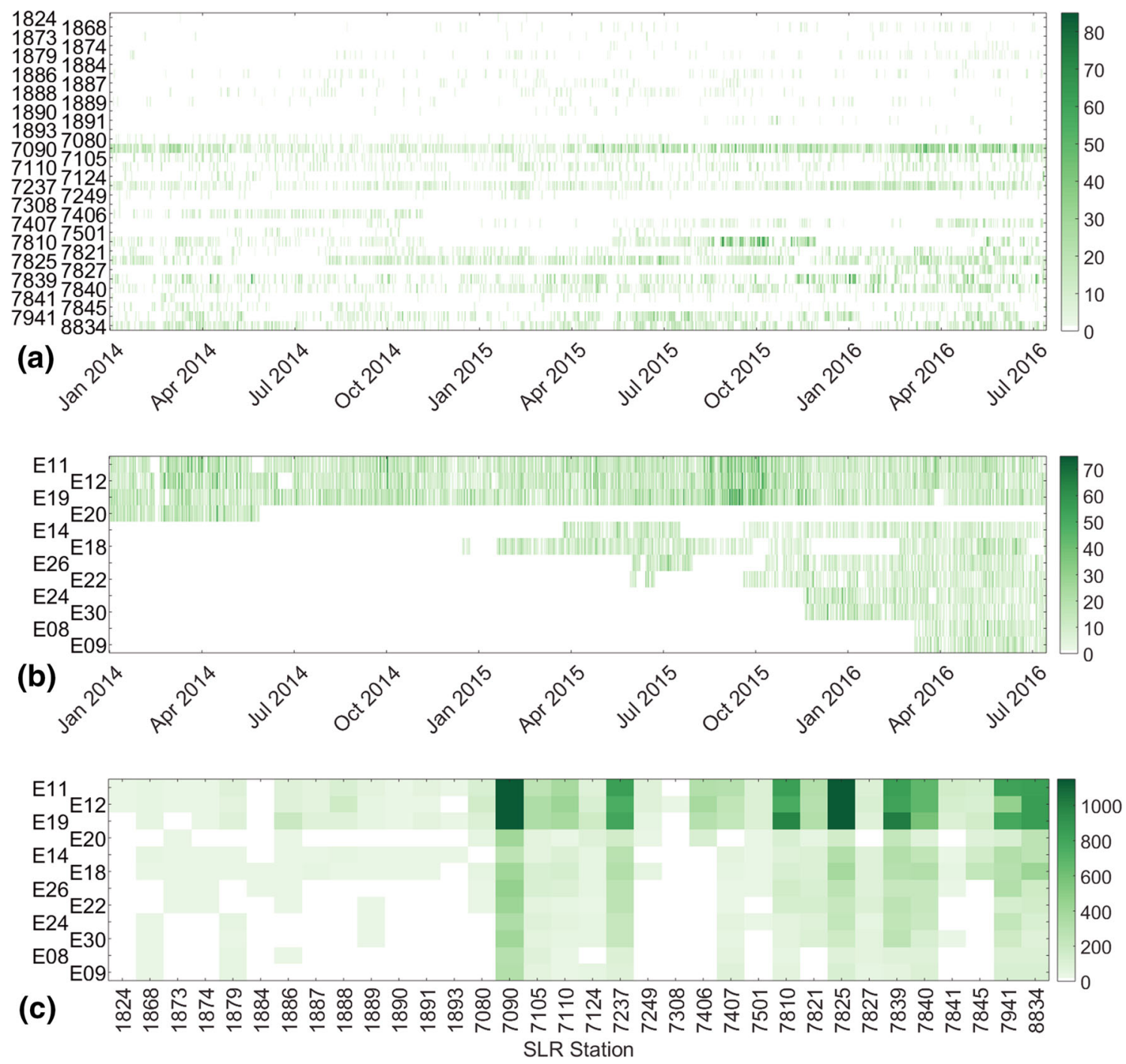

Fig. 3 Daily statistics of SLR observations to Galileo satellites: a number of SLR observations to all Galileo satellites collected by SLR stations as a function of time; $\mathbf{b}$ number of SLR observations to specific
Galileo satellites as a function of time; $\mathbf{c}$ number of SLR observations to specific Galileo satellites as a function of SLR stations

only on one frequency E1 since September 2014 due to a serious power outage. Therefore, the CODE MGEX orbits are not generated for this satellite as the application of the ionosphere-free linear combination is not possible and the SLR validation is also not performed after 2014 due to missing microwave-based orbits. Plane A is occupied by 4 FOC satellites: E24 and E30 launched in September 2015, and E01, E02, launched in May 2016, which are still (August 2016) in the commissioning phase.

Satellites E18 and E14 form another orbital plane, whose orientation changes with respect to the other Galileo orbital planes due to a different revolution period of the ascending node, i.e., 26 years instead of 37 years. The shorter nodal revolution period is caused by the smaller semi-major axis, larger eccentricity, and most importantly, the lower inclina- 


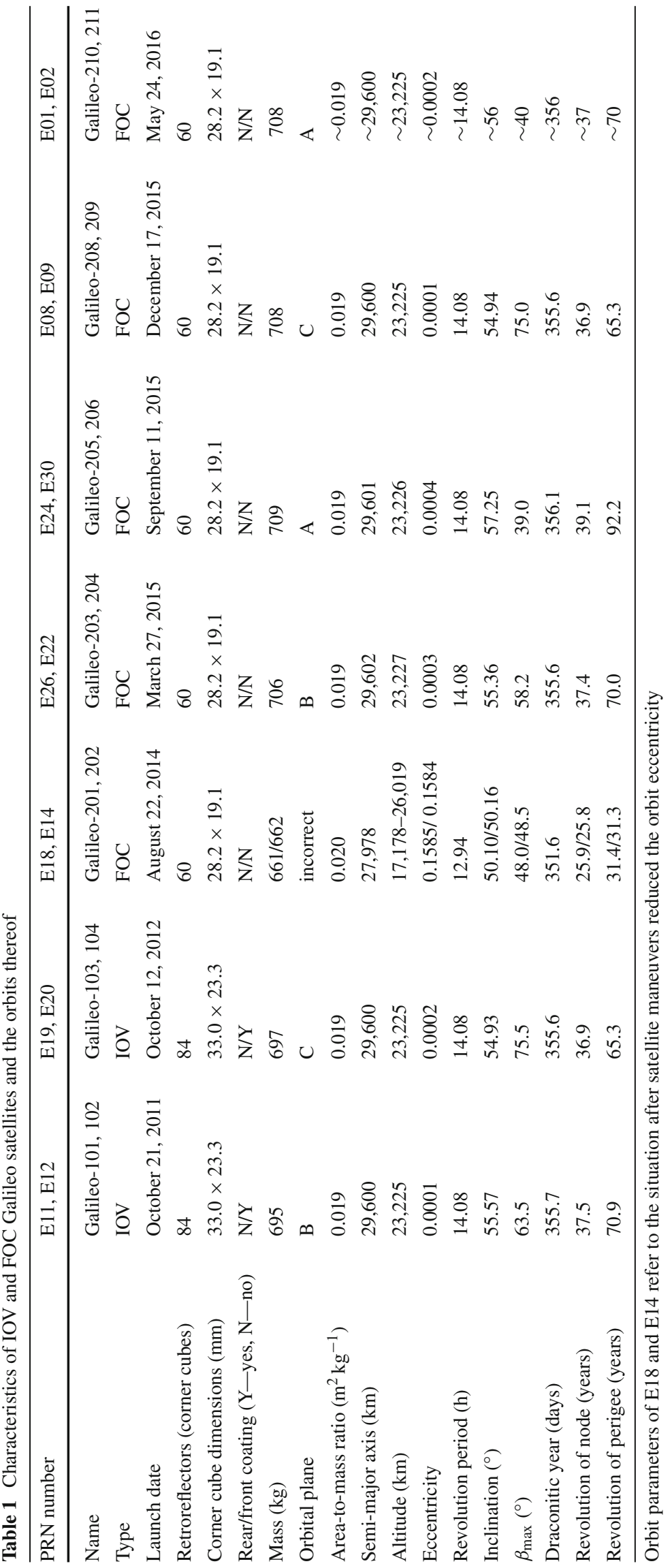




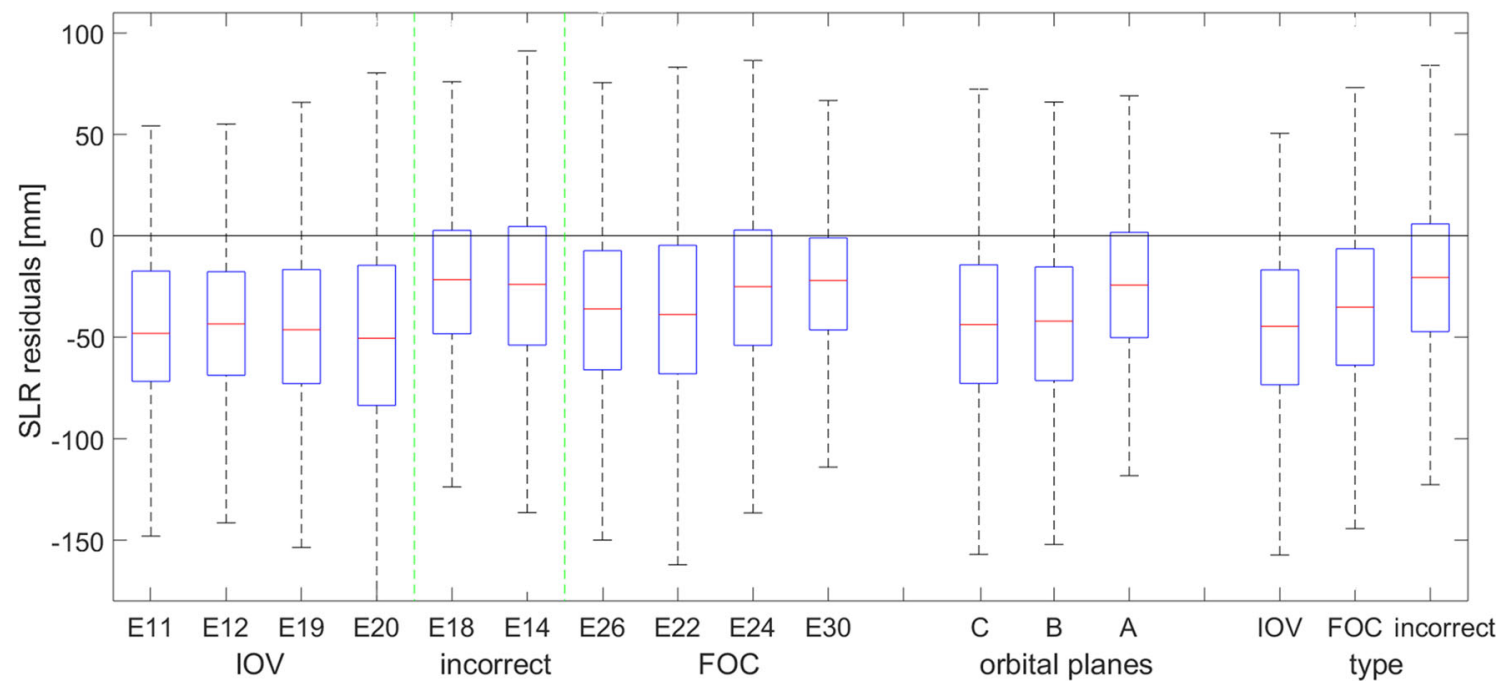

Fig. 4 Median values and interquartile ranges of SLR residuals for individual Galileo satellites, different orbital planes, and different satellite types

tion angle. The satellites complete 13 orbit revolutions in 7 days. The masses of E18 and E14 are smaller by about $46 \mathrm{~kg}$ with respect to other FOC satellites, due to the fuel loss expended on orbit maneuvers when reducing the orbital eccentricity after the launch. The smaller mass results in increase in the area-to-mass ratio by $7 \%$ and thus also in a larger sensitivity to non-gravitational orbit perturbations. The draconitic year of E18 and E14 is about 351.6 days, which is very similar to that of GLONASS orbits. Most of the deficiencies in GNSS orbit modeling appear as spurious variations of the draconitic year period or its harmonics in the GNSSderived parameters (Rodriguez-Solano et al. 2014; Lutz et al. 2016). Draconitic years for nominal and abnormal Galileo orbits differ only slightly (by 4 days), which will not be very helpful in separating the draconitic from geophysical signals in the GNSS-derived parameters.

The maximum $|\beta|$ angle is relatively low for the incorrect orbital planes, equaling to $48^{\circ}$. The orbit modeling for eclipsing satellites and satellites at low $|\beta|$ is typically most challenging, as the Sun illuminates the three surfaces of the satellite body $(+Z,+X$, and $-Z),{ }^{4}$ the illuminated crosssectional area varies most, and the satellite has to slew quickly in order to keep the proper yaw orientation. Finally, one has to keep in mind that neither ECOM1 nor ECOM2 were designed for the solar radiation pressure modeling of the non-circular GNSS orbits or attitude modes deviating from strict yaw attitude, which happens when dynamic yaw steering is applied during deep eclipses.

\footnotetext{
${ }^{4}$ The axis naming is here consistent with the IGS conventions; thus, ' $+X$ ' means ' $+X_{\mathrm{IGS}}$ ' but not ' $+X_{\text {Galileo.' }}$ For details, see Montenbruck et al. (2015a).
}

\section{Validation of Galileo orbits}

This section evaluates the quality of Galileo orbits as a function of orbital planes, satellite types, and $|\beta|$ angles (see Fig. 2). Here, all SLR stations are taken into account without a distinction between the stations equipped with different detector types.

\subsection{Mean offsets and the RMS of SLR residuals}

Figure 4 and Table 2 provide the statistics for individual Galileo satellites with the mean and median SLR offsets, mean RMS and interquartile ranges, maximum $|\beta|$ angle for orbital planes (between 2014.0 and 2016.5), as well as the dependency of SLR residuals on the elongation of the Sun, which characterizes the quality of satellite orbits.

The mean offset of SLR residuals is smallest for E18 and E14, i.e., both in incorrect orbital planes and for E24 and E30, i.e., recently activated FOC satellites. For these satellites, the mean offset is between -22 and $-26 \mathrm{~mm}$, whereas for remaining Galileo satellites the mean offset is in the range between -36 and $-49 \mathrm{~mm}$. The largest offset and the largest RMS are for E20, i.e., an IOV satellite with the microwave GNSS solutions only in 2014, as it has been transmitting the signal only on one frequency since 2014. In 2014, the ground network of IGS MGEX stations tracking the Galileo signal was inferior as compared to the situation from 2015 and 2016, which is related to the poorer quality of E20 orbits. The RMS of residuals is above $40 \mathrm{~mm}$ for most satellites, except for E30, E18, and E12. E30 is a new satellite with a relatively small number of SLR observations; E18 is a satellite launched into incorrect orbit, whereas E12 is an IOV satellite with a relatively large number of SLR observations at high $|\beta|$ 
Table 2 Characteristics of IOV and FOC Galileo satellites and the orbits thereof

\begin{tabular}{lccccc}
\hline Satellite (plane) & $\begin{array}{l}\text { No of } \\
\text { observations }\end{array}$ & $\begin{array}{l}\text { Mean offset } \\
(\mathrm{mm})\end{array}$ & RMS* $(\mathrm{mm})$ & $\beta_{\max }\left(^{\circ}\right)$ & $\begin{array}{l}\text { Elongation } \\
\text { slope }\left(\mathrm{mm} /{ }^{\circ}\right)\end{array}$ \\
\hline E11 (B) & 11,403 & -46.4 & 41.9 & 63.5 & -0.58 \\
E12 (B) & 10,692 & -44.5 & 37.6 & 63.5 & -0.23 \\
E19 (C) & 11,427 & -43.2 & 42.7 & 75.5 & -0.62 \\
E20 (C) & 1888 & -49.2 & 50.8 & 72.5 & -0.91 \\
E18 (incorrect) & 3403 & -22.1 & 38.7 & 48.0 & -0.44 \\
E14 (incorrect) & 2611 & -22.9 & 41.3 & 48.5 & -0.41 \\
E26 (B) & 2338 & -36.6 & 46.1 & 58.1 & -0.81 \\
E22 (B) & 1934 & -38.6 & 47.1 & 58.2 & -0.90 \\
E24 (A) & 583 & -26.2 & 40.1 & 39.0 & -0.67 \\
E30 (A) & 489 & -23.9 & 35.4 & 39.0 & -0.52 \\
Orbital plane & & & & & \\
A & 1072 & -25.2 & 38.0 & 39.0 & -0.60 \\
B & 26,367 & -44.2 & 40.9 & 62.6 & -0.48 \\
C & 13,315 & -44.1 & 43.8 & 75.1 & -0.66 \\
Satellite type & & & & & \\
IOV & 35,410 & -44.9 & 41.3 & 67.9 & -0.50 \\
FOC & 5344 & -35.0 & 44.8 & 54.3 & -0.80 \\
FOC incorrect & 6014 & -22.4 & 48.2 & -0.43 \\
\hline Orbit parameter & & & & & \\
\hline
\end{tabular}

Orbit parameters of E18 and E14 refer to the situation after satellite maneuvers correcting the orbit eccentricity * RMS denotes here a standard deviation after removing the mean value angles. The RMS values of SLR residuals are smaller when considering data only from selected high-performing stations as given in Table 3 (see discussion in Sect. 5).

When comparing different orbital planes, one would expect that for the planes with lower $\beta_{\max }$, the RMS of SLR residuals should be larger due to solar radiation pressure modeling issues. However, this is not the case, as the orbital plane A has the smallest RMS and the smallest mean offset of 28 and $-25 \mathrm{~mm}$, respectively, despite having the lowest $\beta_{\max }$. The orbital plane A has recently been populated; thus, the quality of microwave Galileo orbits is high due to a good distribution of the GNSS ground observing network. For planes C and B, the mean offset is $-44 \mathrm{~mm}$ with the RMS of about $42 \mathrm{~mm}$.

From the comparison between IOV, FOC, and FOC in incorrect orbits, the RMS is 41,45 , and $40 \mathrm{~mm}$ and the mean offset is $-45,-35$, and $-22 \mathrm{~mm}$, respectively. The satellites in incorrect orbits have reduced masses by $46 \mathrm{~kg}$, due to the fuel loss that was needed for maneuvers which corrected the orbital eccentricity. This raises the question of whether the different offsets in the radial direction, which coincides with the $Z$ axis of the satellite, can be explained by the displacement of the satellite center-of-mass. ESA has published time-dependent center-of-mass values for all Galileo satellites. ${ }^{5}$ According to this information, the difference of the

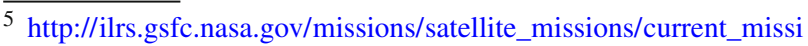
ons/ga01_com.html.
}

Table 3 Mean offsets, RMS, and sigma of SLR observations residuals (calculated w.r.t. zero value) for SLR stations equipped with different detector types

\begin{tabular}{lllll}
\hline Stations & $\begin{array}{l}\text { No of } \\
\text { observations }\end{array}$ & $\begin{array}{l}\text { Mean offset } \\
(\mathrm{mm})\end{array}$ & $\begin{array}{l}\text { RMS } \\
(\mathrm{mm})\end{array}$ & $\begin{array}{l}\text { Sigma } \\
(\mathrm{mm})\end{array}$ \\
\hline All MCP & 20,900 & -41.1 & 38.4 & 56.3 \\
$\begin{array}{l}\text { MCP excluding } \\
\quad \text { Tahiti }\end{array}$ & 20,376 & -43.4 & 38.2 & 58.2 \\
$\begin{array}{l}\text { All C-SPAD } \\
\text { C-SPAD excluding }\end{array}$ & 21,507 & -38.9 & 41.6 & 56.9 \\
$\quad$ Changchun, San & 16,806 & -29.5 & 35.1 & 45.9 \\
$\quad \begin{array}{l}\text { Juan, Beijing } \\
\text { All PMT }\end{array}$ & & & & \\
$\begin{array}{l}\text { PMT excluding } \\
\text { Komsomolsk }\end{array}$ & 2877 & -41.1 & 54.0 & 67.9 \\
\hline
\end{tabular}

center-of-mass for Galileo E18 between November 2014 and February 2016 is $3.0,0.0$, and $0.0 \mathrm{~mm}$ for the $X, Y$, and $Z$ components, whereas the difference between E18 and other FOC amounts to about $57.4,4.2$, and $0.8 \mathrm{~mm}$ for the $X, Y$, and $Z$ components, respectively. This means that the fuel consumption changes the satellite center-of-mass mostly along the $X$ axis, whereas the $Z$ axis pointing to the Earth and lying along the radial direction, i.e., the direction of the largest sensitivity to SLR observations, changes only insignificantly. In no case can the reduced satellite mass account for the different SLR offset of the order of 13-22 $\mathrm{mm}$. 


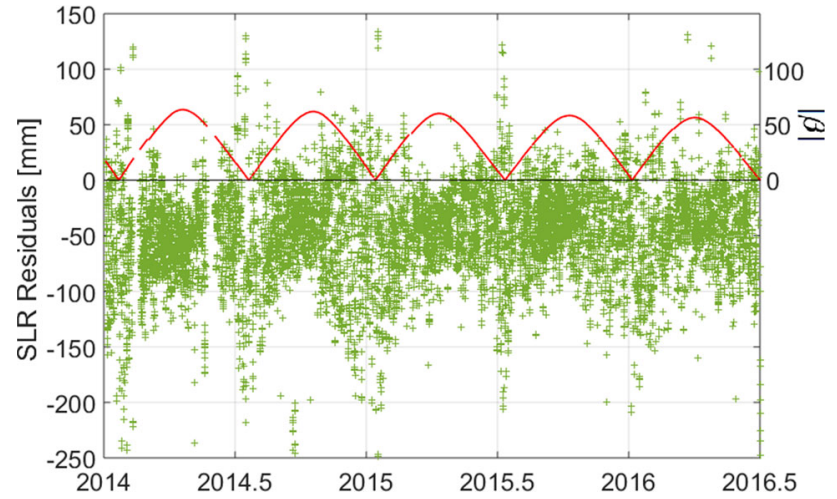

Fig. 5 SLR observation residuals to Galileo E11 as a function of time and the Sun elevation angle $|\beta|$ above the orbital plane

We consider it likely that the SLR offsets shown in Fig. 4 might well be reduced through a proper modeling of the Earth radiation pressure and the transmitter antenna thrust. For example, for GPS and GLONASS, the albedo modeling reduces the offset by about $10 \mathrm{~mm}$, whereas the antenna thrust reduces the offset further by $5-10 \mathrm{~mm}$ (RodriguezSolano et al. 2012; Fritsche et al. 2014). Steigenberger et al. (2017) found that the missing antenna thrust accounts for a radial offset of 15 and $26 \mathrm{~mm}$ for IOV and FOC, respectively, and 17-32 mm for FOC in incorrect orbits depending whether the satellites are in perigee or apogee. Using the a priori solar radiation pressure model may also reduce the SLR offset (Steigenberger and Montenbruck 2017).

\subsection{Orbit modeling issues}

Figure 5 shows the time series of SLR residuals to E11 and the absolute value of the Sun elevation angle $|\beta|$ above the orbital plane. The Sun crosses through the orbital plane every 177.8 days, whereas the passage in the same direction takes place every 355.6 days, i.e., once per draconitic year. The maximum beta angle changes over time due to a slow precession of the orbital plane, whose full revolution lasts on average 37 years (see Table 1 ).

Despite using the improved model ECOM2, which substantially reduces the systematic $\beta$-dependent effects, the spread of SLR residuals is still much larger for the lowest $|\beta|$ angles. This is on the one hand due to the orbit modeling deficiencies and on the other hand due to the dynamic yaw steering of Galileo satellites for $|\beta|<2^{\circ}$ (Ebert and Oesterlin 2008).

\subsection{Dependency on satellite-Sun elongation angle}

Now, we examine the SLR residuals as a function of the satellite-Sun elongation angle $\varepsilon$ and the satellite latitude $\Delta u$ w.r.t. the Sun that are defined as (see Fig. 2):
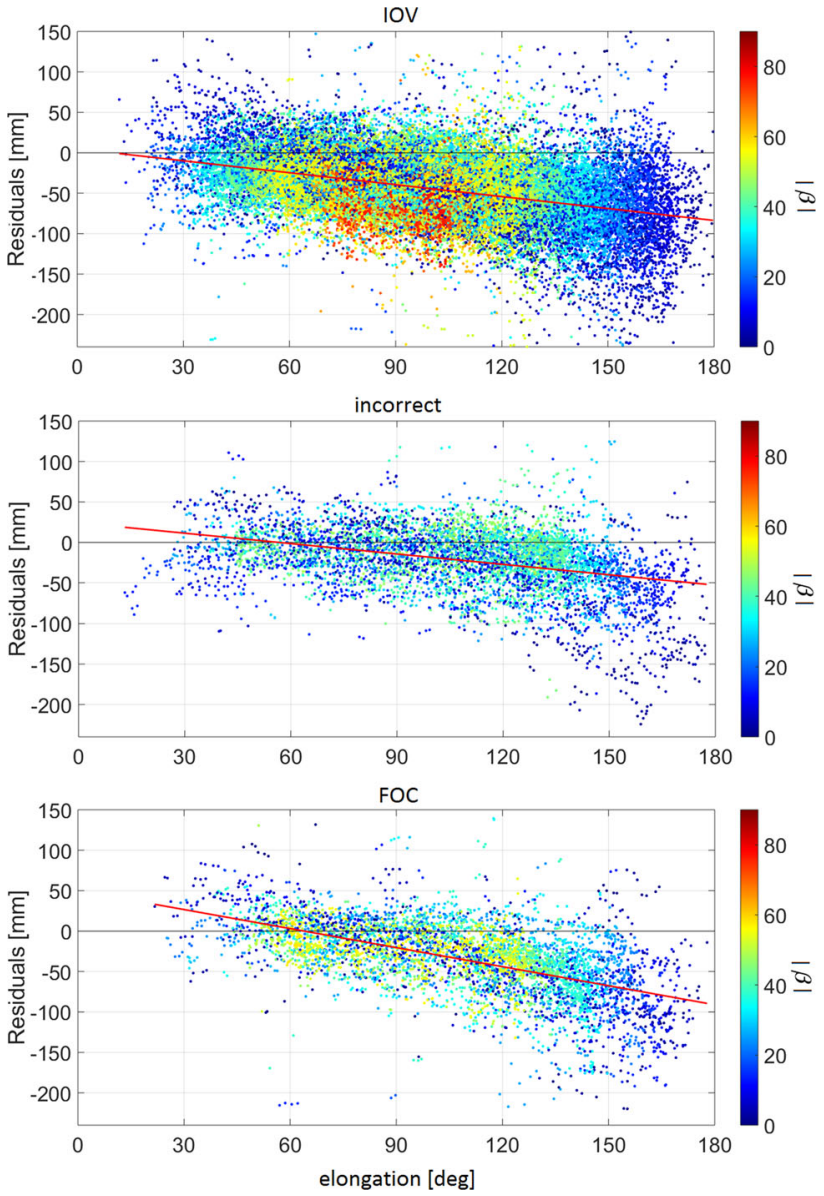

Fig. 6 Dependency of SLR residuals on the satellite elongation angle w.r.t. the Sun when using ECOM2

$\cos \varepsilon=\cos \beta \cos \Delta u$.

Figure 6 shows that all satellite types, IOV, FOC incorrect, and FOC, exhibit a residual dependency on the elongation angle. For high $|\beta|$ (red and yellow dots in Fig. 6), the elongation angle is close to $90^{\circ}$ and the spread of SLR residuals becomes much smaller than for low $|\beta|$ having typically a large dispersion (blue dots in Fig. 6). When the $|\beta|$ angle is high, the Sun illuminates the $+X$ surface of the satellite box for most of the time $\left(+X_{\mathrm{IGS}}\right.$ in Fig. 9 from Montenbruck et al. 2015a). For low $|\beta|$ angles, the Sun illuminates $+X,+Z$, and $-Z$ sides, which results in large temporal variations of the surface area exposed to the Sun for elongated satellite bodies, and thus causes difficulties in a proper solar radiation pressure modeling. The twice-per-revolution components in the $\mathrm{D}$ direction of the new ECOM2 were added to absorb in particular the effect of the temporal exposure variability (Arnold et al. 2015).

The mean slope of the elongation dependency is -0.50 , -0.43 , and $-0.80 \mathrm{~mm} /{ }^{\circ}$ for IOV, incorrect, and FOC satellites, respectively (see Fig. 6). The smallest slope results for 
incorrect orbital planes, despite that the empirical orbit models were elaborated for circular and not for highly eccentric satellite orbits with variable angular and linear velocities. For the incorrect orbital planes, $\beta_{\max }$ is relatively low as compared to IOV and FOC, and does not exceed $48^{\circ}$, which should cause a higher sensitivity of these planes to orbit modeling deficiencies. Despite lower $|\beta|$, smaller masses, and larger area-to-mass ratios, the orbit quality of Galileo satellites in incorrect planes is remarkably good with the smallest slope of the elongation dependency when compared to other Galileo satellites. High orbit quality of Galileo satellites in incorrect orbits is not only essential for precise geodetic and geophysical applications, but also is a prerequisite for the test of the gravitational redshift employing stable Galileo orbits and clocks (Delva et al. 2015).

Prange et al. (2017) found that the interquartile ranges of orbit misclosures in the radial direction are 15 and $11 \mathrm{~mm}$ for IOV and FOC in incorrect orbits when using ECOM2, and 38 and $34 \mathrm{~mm}$ when using ECOM1, respectively. Smaller orbit misclosure errors in the radial direction for the incorrect orbits confirm again the high quality of Galileo orbits in the incorrect planes. On the other hand, the quality of the alongtrack component is inferior for the incorrect orbits with a median discontinuity of $26 \mathrm{~mm}$ when compared to $3 \mathrm{~mm}$ discontinuity of IOV (Prange et al. 2017) and it may even increase when the 1-day satellite arcs are generated instead of 3-day arcs. However, SLR is not sensitive to the alongtrack orbit errors; thus, SLR residuals do not demonstrate any orbit misclosure issues in along-track.

\section{SLR signature effect}

The SLR stations typically employ 3 different detector types:

- Micro-Channel Plate (MCP),

- Photo-Multiplier Tube (PMT),

- Compensated Single-Photon Avalanche Diode (C-SPAD).

MCP and PMT detectors have the capability of registering many photons and thus belong to the type of high-energy detectors. SPAD detectors register single to a few photons with the fast rise time of the avalanche to give good epoch timing and high stability. The improved version of the SPAD detectors is the C-SPAD with a better temperature stabilization and the compensation for time walk. However, in single-photon detectors for each firing of the laser only a single event can be recorded by the C-SPAD, which means that the detector may instead register background noise or daylight as an observation. Noise is reduced by close gating, small fields of view and narrow spectral filters tuned to the laser wavelength.

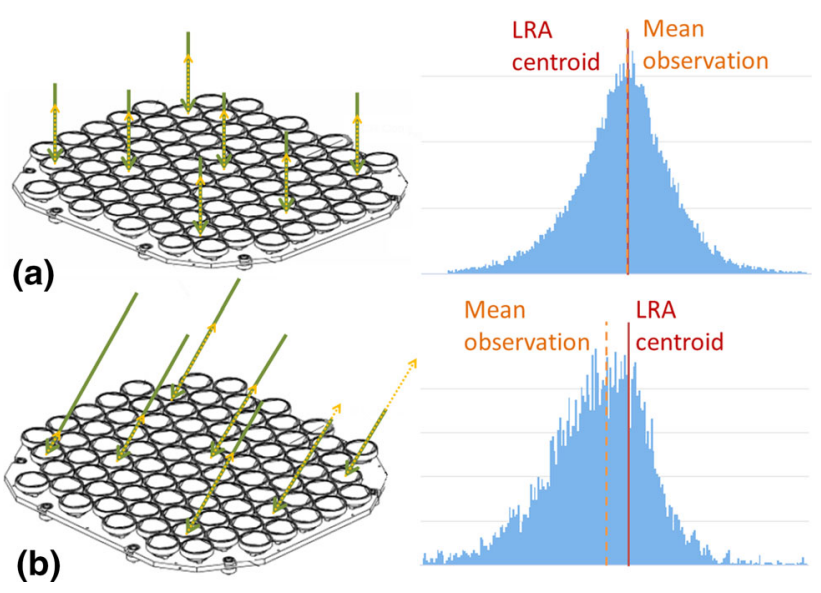

Fig. 7 Satellite signature effect for multi-photon detectors: a the nadir angle is 0 and the LRA centroid coincides with the mean observation, $\mathbf{b}$ the LRA is inclined and the detection timing is defined at some threshold level at the leading edge of the return pulse; as a result, the mean registered range is shorter than the actual one

Most of the NASA SLR stations are equipped with highenergy MCP detectors, e.g., Monument Peak (7110, USA), Greenbelt (7105, USA), McDonald (7080, USA), Yarragadee (7090, Australia), Tahiti (7124, French Polynesia), as well as Matera (7941, Italy) and Wettzell (8834, Germany). The PMT detectors are typically employed by the Russian stations, such as: Arkhyz (1886), Svetloe (1888), Altay (1879), Zelechukskaya (1889), Baikonur (1887), Komsomolsk (1868, all in Russia), Brasilia (7407, Brazil), as well as by Hartebeesthoek (7501, South Africa). SPAD or CSPAD detectors are used by the majority of the European SLR stations: Herstmonceux (7840, UK), Graz (7839, Austria), Zimmerwald (7810, Switzerland), Grasse (7845, France), as well as by recently upgraded Chinese stations: Changchun (7237), Beijing (7249), Shanghai (7821, all in China), San Juan (7403, Argentina), and by Mt Stromlo (7825, Australia). Some SLR stations are equipped with multiple detectors, e.g., PMT and C-SPAD in Potsdam (7841, Germany).

MCP and PMT detectors may be affected by the satellite signature effect when the energy is not properly controlled. The satellite signature effect is defined as a spread of optical pulse signals due to reflection from multiple reflectors, which causes variations of mean SLR residuals as a function of satellite's nadir angle (Otsubo et al. 2001). The effective array size, which is the measure of the spread of optical pulse signals due to the reflection from multiple reflectors, is higher for high-energy detection systems, because the detection timing is defined at some threshold level at the leading edge of the return pulse. When the flat LRA is inclined, and not perpendicular with respect to the observer, the first response comes from the nearest edge of the LRA, and thus, the ranges registered are shorter when the threshold level does not allow registering all incoming photons (see Fig. 7). 


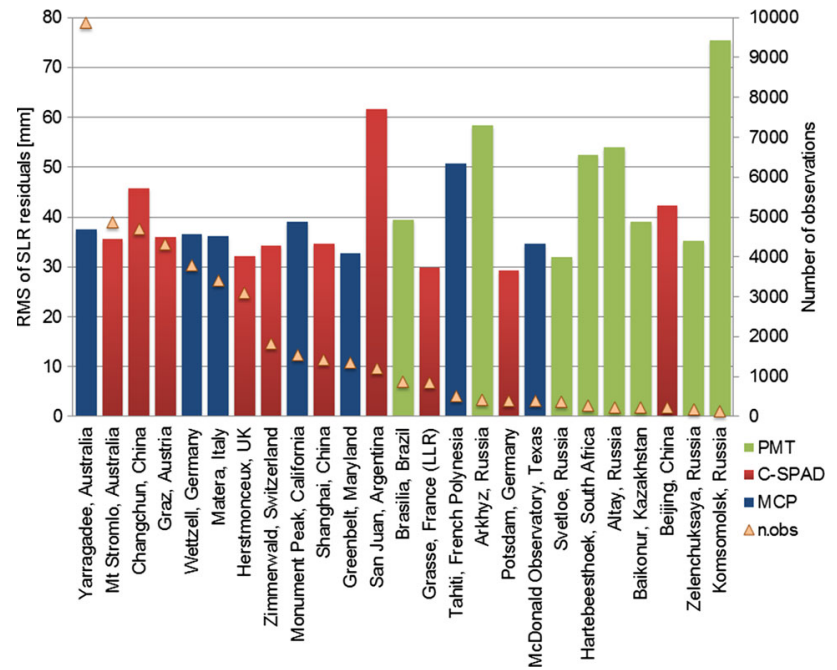

Fig. 8 RMS of SLR residuals and the number of observations to Galileo collected by different SLR stations

In the single-photon systems, operating in the low-energy mode, the probability of a photon reflection is the same for every corner cube, and thus, the average reflection point, after several hundreds of reflections, coincides with the center of LRA which in turn corresponds to the centroid of the residual histogram. As each detected photon may originate from any of the retroreflectors, the spatial distribution of the whole array is mapped over many detections (Otsubo et al. 2001). Thus, the SLR stations operating in the singlephoton mode should not be affected by the issues related to different incidence angles of a laser beam for flat LRAs. However, the single-photon stations require post-processing rejection of high-rate data and responsive energy control by signal reduction filtering when tracking at different elevation angles, because the optical path of a laser pulse through the troposphere varies substantially between the zenith and lowelevation observations, thus leading to intrinsically variable return signal strengths. In addition, the signal variability during a pass may be caused by the telescope and laser pointing, either due to imperfect tracking or variability due to atmospheric turbulence.

Figure 8 shows the RMS of SLR observation residuals to all Galileo satellites with a distinction between the stations equipped with different detector types. For most of the SLR stations, the RMS values are between 29 and $39 \mathrm{~mm}$. The smallest RMS is obtained for stations, Potsdam, Grasse, and Herstmonceux, and equals to 29, 30, and $32 \mathrm{~mm}$, respectively. All of these stations are equipped with C-SPAD or SPAD detectors or operate in the low-energy return rate regime (Potsdam). PMT stations are typically characterized by larger RMS than MCP and C-SPAD stations. This can be related to the fact that the a priori station coordinates in SLRF2008 are based only on a relatively short time series for newly estab- lished stations, e.g., Arkhyz, Altay, Brasilia, and that some PMT stations are characterized by a high RMS of singleshot observations, e.g., Komsomolsk. The mean RMS of SLR residuals for PMT stations is $54.0 \mathrm{~mm}$ for all PMT stations and $51.2 \mathrm{~mm}$ when excluding Komsomolsk, respectively (see Table 3). For MCP stations, the mean RMS of SLR residuals is $38.4 \mathrm{~mm}$ for all stations and $38.2 \mathrm{~mm}$ when excluding the Tahiti station which is characterized by the highest RMS value and employs interval timers, whereas most of remaining stations upgraded already to event timers. The RMS values for C-SPAD detectors do typically not exceed $40 \mathrm{~mm}$, except for three Chinese stations: Changchun, San Juan, and Beijing. The mean RMS values for all C-SPAD stations is $41.6 \mathrm{~mm}$ for all stations and $35.1 \mathrm{~mm}$ when excluding three Chinese stations (see Table 3).

The mean offset equals to $-43.4,-43.9$, and $-29.5 \mathrm{~mm}$ for selected MCP, PMT, and C-SPAD stations, respectively (see Table 3). The difference of $14 \mathrm{~mm}$ between the offsets for multi-photon and single-photon stations is mostly due to the satellite signature effect. The remaining offset is most likely due to orbit modeling deficiencies, in particular the missing transmitter antenna thrust and Earth radiation pressure models.

\subsection{Dependence on the incident angle}

Figure 9 shows four examples of the dependence of the SLR observation residuals on the incident angle of a laser beam. Wettzell is a good representative of all high-energy stations equipped with MCP or PMT: The offset in the nadir direction is about $-20 \mathrm{~mm}$ and the slope w.r.t. nadir angle is about $-2.0 \mathrm{~mm} /{ }^{\circ}$. The slope is a consequence of the satellite signature effect due to the first reflection of laser pulses from the nearest edge of the flat retroreflector. Herstmonceux is one of the SLR stations operating strictly in the single-photon regime. For Herstmonceux and for most of the C-SPAD stations, the nadir offset equals to $-20 \mathrm{~mm}$, whereas the slope is slightly smaller than that for high-energy stations and is about $-1.5 \mathrm{~mm} /{ }^{\circ}$. The slope for C-SPAD detectors is quite large when compared, e.g., to the GLONASS-M satellites equipped with uncoated corner cubes, for which the slope did not exceed $0.1 \mathrm{~mm} /{ }^{\circ}$. The larger slope may suggest the existence of intensity-dependent bias or some orbit modeling issues as the quality of Galileo orbits is still slightly worse than the GLONASS-M orbits, i.e., the RMS of SLR residuals is 32 and $36 \mathrm{~mm}$ for GLONASS-M with uncoated and coated retroreflectors, respectively (Sośnica et al. 2015).

Figure 9 shows examples of two single-photon stations with an anomalous pattern as compared to other singlephoton stations. Changchun has a large negative offset in the nadir direction of $-93 \mathrm{~mm}$ and the slope of $+2.6 \mathrm{~mm} /{ }^{\circ}$, whereas Grasse, equipped with an uncompensated SPAD, has an offset of $-66 \mathrm{~mm}$ and the slope of $+2.3 \mathrm{~mm} /{ }^{\circ}$. The 
Fig. 9 Dependency of SLR residuals on the incidence angle of the laser beam with respect to flat LRAs onboard Galileo satellites
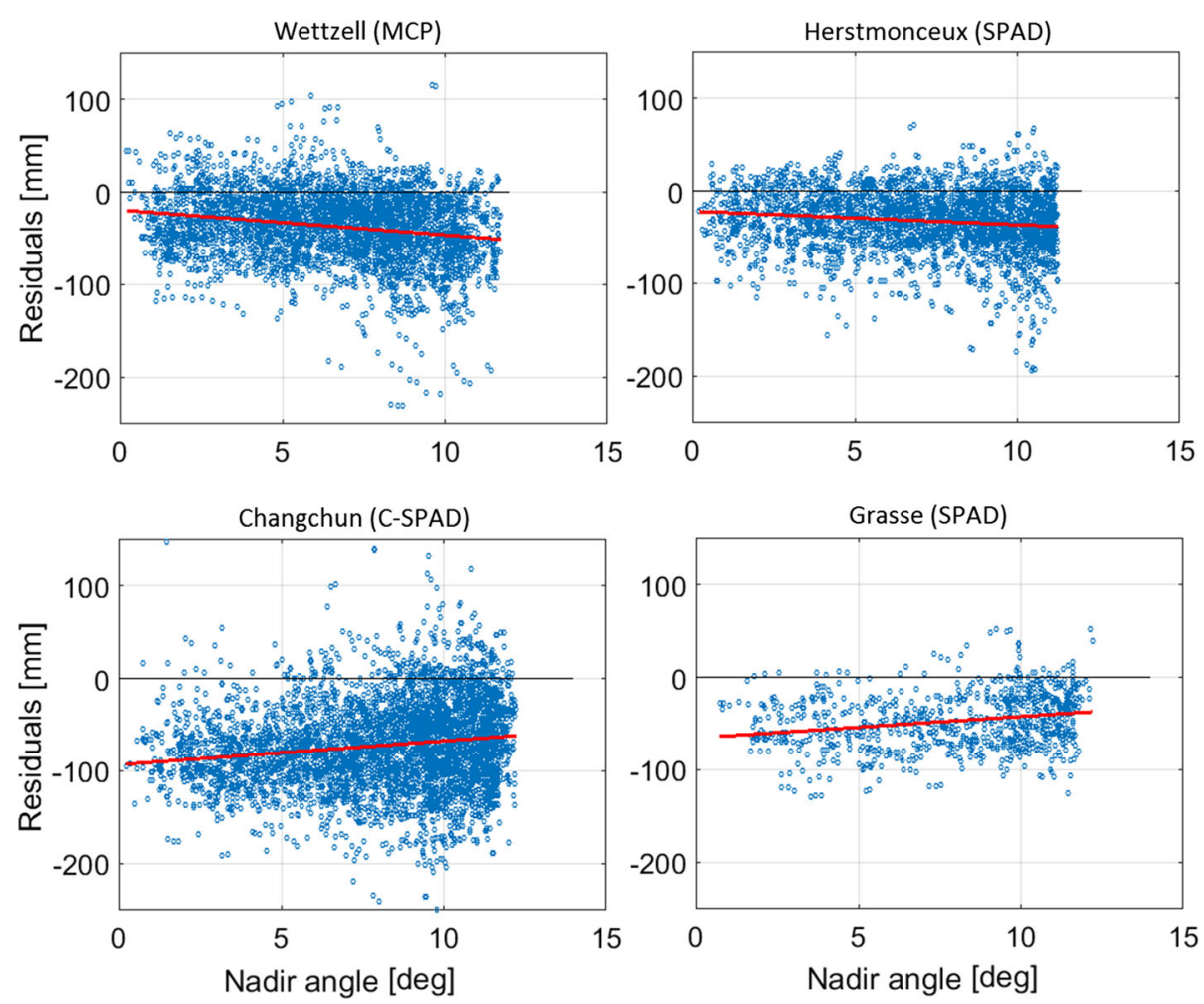

slope is about three times larger than previously observed for C-SPAD stations tracking GLONASS-M with coated corner cubes (Sośnica et al. 2015). However, the Galileo satellites have no coating on the rear side of the corner cubes and only the front side of IOV is coated with an anti-reflection layer. Therefore, the anomalously large positive slope rather suggests that there may be some issues with proper background noise filtering or intensity-dependent bias at these SPAD stations. Moreover, Dong et al. (2016) reported recently discovered issues with the stability of temperature around the Changchun station.

\subsection{IOV, FOC, and Galileo satellites in incorrect orbits}

The SLR residuals are a function of the detector type as well as the retroreflector type. The LRA installed on IOV satellites comprises 80 corner cubes of a size of $33.0 \times 23.3 \mathrm{~mm}$, whereas FOC satellites carry LRA with 60 corner cubes of a size of $28.2 \times 19.1 \mathrm{~mm}$. LRAs were not designed to be carried on satellites in eccentric orbits whose angular velocity is not constant during a single pass. However, the velocity aberration problems as seen for lunar laser ranging (Otsubo et al. 2011) should not be directly observable, as the SLR is mostly sensitive to the radial orbit direction. Nevertheless, all these issues may introduce different characteristics for different satellite types and detector types.

Figure 10 shows the SLR residuals for IOV, FOC, and satellites in incorrect orbits collected by selected stations employing MCP, C-SPAD, and PMT detectors. The selected MCP stations include: Yarragadee, Wettzell, Matera, Monument Peak, Greenbelt, and McDonald. The C-SPAD stations include Herstmonceux operating strictly in the single-photon mode, Graz and Zimmerwald collecting one to a few photons and Potsdam operating in the low-energy regime. All of these C-SPAD stations operate in at least $100 \mathrm{~Hz}$ mode (Zimmerwald) or $\mathrm{kHz}$ mode and employ event timers. PMT stations include Hartebeesthoek and all the stations from the Russian network except for Komsomolsk.

The RMS of SLR residuals is largest for PMT stations and amounts between 48 and $89 \mathrm{~mm}$ for IOV and FOC, respectively (see Table 4). The number of collected SLR observations is also remarkably smaller for PMT stations as compared to MCP and C-SPAD, despite that this group includes the largest number of SLR stations.

Interestingly, the smallest offset amounting to only $-6.7 \mathrm{~mm}$ is obtained for C-SPAD detectors when tracking Galileo in incorrect orbits (see Table 4). The RMS is at the level of $23 \mathrm{~mm}$, which is to some extent similar to the quality of SLR observations to historical GPS-35/36 satellites (offset: -12 mm, RMS: 23 mm) (Sośnica et al. 2015). However, only few of these observations were collected for $|\beta|$ angles lower than $2^{\circ}$, whereupon the solar radiation pressure modeling is more demanding and the dynamic yaw steering is not entirely handled in a proper way. The smallest RMS of SLR residuals to incorrect Galileo orbits is 22.3, 23.0, and $25.1 \mathrm{~mm}$ for Graz, Herstmonceux, and Zimmer- 

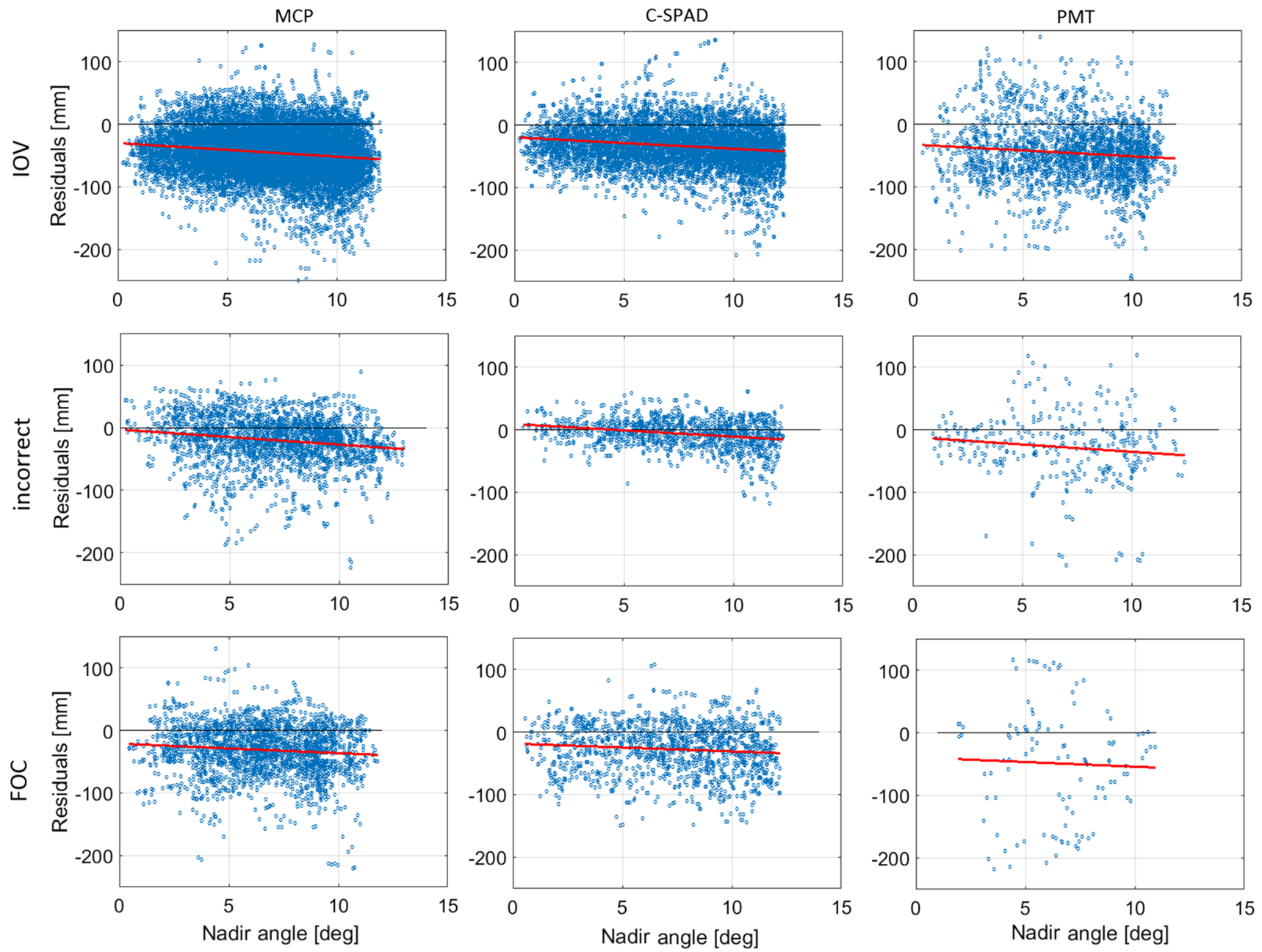

Fig. 10 SLR observation residuals for IOV, FOC, and incorrect Galileo orbits as well as for MCP, C-SPAD, and PMT detectors

wald, respectively. The RMS for Potsdam tracking incorrect Galileo is only $8.6 \mathrm{~mm}$; however, the number of observations amounts to only 33 ; thus, the RMS calculation may not be fully reliable. The slope dependency between the SLR residuals and nadir angle is rather detector-specific than satellite type-specific (see Table 4).

The FOC satellites tracked by C-SPAD stations have larger offsets by $19 \mathrm{~mm}$ and RMS values larger by $14 \mathrm{~mm}$ than Galileo satellites in incorrect orbits despite the comparable number of observations. Therefore, one can conclude that the quality of Galileo satellite orbits launched into incorrect planes is slightly better than IOV and FOC based on the smaller offset of SLR residuals and a remarkably smaller RMS of residuals for the single-photon detectors.

Figure 10 implies as well that the SLR residuals should rather be analyzed separately for different detector types instead of a collective analysis for all SLR stations. The individual treatment of satellite center-of-mass corrections for every detector type was proposed and implemented for LAGEOS, Etalon, and Ajisai (Otsubo and Appleby 2003) and recently also for LARES (Otsubo et al. 2015) on the basis of simulated detected distributions and target response impulse functions from a range of laser widths, total system noise, and return rate threshold settings. Figure 10 suggests that the GNSS satellites equipped with flat LRAs should also have detector-specific corrections with a properly considered satellite signature effect so that the SLR measurements represent the LRAs' centroid, instead of using one offset value for all SLR stations. The alternative for the current approach may be the detection of the signal incoming from individual LRA corner cubes as proposed by Kucharski et al. (2015) for Ajisai.

\section{The classical and the new ECOM}

In order to evaluate the impact of the a priori empirical orbit model, the operational CODE MGEX products from 2014 generated using ECOM1 are compared to the reprocessed products from 2014 using ECOM2. 
Table 4 Mean offset, RMS, residual dependency on the incident angle, and number of SLR observations collected by selected stations equipped with MCP, C-SPAD, and PMT detectors to IOV, FOC, and Galileo satellites in incorrect orbits

\begin{tabular}{lccc}
\hline & MCP & C-SPAD & PMT \\
\hline Mean offset $(\mathrm{mm})$ & & & \\
IOV & -45.8 & -33.5 & -46.1 \\
Incorrect & -18.6 & -6.7 & -27.0 \\
FOC & -30.0 & -27.7 & -48.8 \\
RMS $(\mathrm{mm})$ & & & \\
IOV & 36.3 & 33.3 & 47.8 \\
Incorrect & 35.7 & 23.0 & 54.3 \\
FOC & 37.3 & 37.2 & 89.1 \\
Slope of SLR residuals $\left(\mathrm{mm} /{ }^{\circ}\right)$ & & \\
IOV & -2.4 & -1.9 & -2.8 \\
Incorrect & -2.1 & -1.0 & -2.5 \\
FOC & -1.7 & -1.2 & -2.1 \\
No. of observations & & & \\
IOV & 15,625 & 7059 & 2314 \\
Incorrect & 2383 & 1238 & 314 \\
FOC & 2329 & 1361 & 110 \\
\hline
\end{tabular}

\subsection{Dependency on the Sun elevation angle}

Figure 11 compares the SLR residuals as a function of the Sun elevation $|\beta|$ and relative Sun-satellite latitude $\Delta u$ angles when using ECOM1 and ECOM2 for all Galileo satellites active and tracked by SLR in 2014. For ECOM1, there is a very strong dependency between SLR residuals and $\Delta u$, especially for $|\beta|<50^{\circ}$. When $\Delta u$ is close to $180^{\circ}$, i.e., when a satellite is in the opposite direction than the Sun as seen from the Earth, the SLR residuals assume the values of about $-200 \mathrm{~mm}$. These large negative SLR residuals do not only occur for eclipsing $\left(|\beta|<12.3^{\circ}\right)$, but for all Galileo satellites when $\Delta u \approx 180^{\circ}$. On the contrary, when $\Delta u \approx 0^{\circ}$ or $360^{\circ}$, the SLR residuals assume maximum values of about $+150 \mathrm{~mm}$. The residuals would almost certainly be even larger if the SLR stations were able to track satellites close to the Sun, i.e., when $\Delta u \approx 0^{\circ}$ and $\beta \approx 0^{\circ}$. This is, however, impossible for optical satellite measurements.

The pattern of the latitude dependency for Galileo satellites using ECOM1 is similar to that observed for GPS Block IIA satellites and opposite to that of GLONASS from Fritsche et al. (2014) (Fig. 10). This is due to different surface areas for the $X$ and $Z$ sides of the satellite body. The surface area of $X$ and $Z$ is 2.7 and 2.9; 4.2 and 1.7; and 1.3 and $3.0 \mathrm{~m}^{2}$; for GPS-IIA, GLONASS, and Galileo, respectively (Flohrer et al. 2015). When the $Z$ surface area is larger than $X$, as in the case of GPS-IIA and Galileo, the SLR residuals assume negative values for $\Delta u \approx 180^{\circ}$. On the contrary, for the $X$ area larger than $Z$, the SLR residuals assume positive val- ues for $\Delta u \approx 180^{\circ}$, as in the case of GLONASS (Fig. 8 in Fritsche et al. 2014).

The switch from ECOM1 to ECOM2 remarkably reduces the spurious SLR residual dependency on the $\Delta u$ angles (see Fig. 11, bottom). However, the systematic pattern is not completely eliminated, as the residuals are about -65 , and $-15 \mathrm{~mm}$ for $\Delta u \approx 180^{\circ}$, and $\Delta u \approx 0^{\circ}$, respectively. Moreover, large negative SLR residuals occur for $|\beta|<8^{\circ}$ and $\Delta u \approx 150^{\circ}$. This can partly be explained by the unmodelled dynamic yaw steering (Ebert and Oesterlin 2008). However, dynamic yaw steering should be visible for $|\beta|<2^{\circ}$, whereas it seems that it is employed already for higher $|\beta|$ angles. Larger residuals could also be explained by the eclipsing seasons, but the satellites enter the shadow already for $|\beta|<13^{\circ}$, whereas increased residuals are not visible for $8^{\circ}<|\beta|<13^{\circ}$. This issue requires thus further investigations.

An alternative improvement of Galileo orbits, instead of using ECOM2, could be obtained through using an adjustable box-wing model (Rodriguez-Solano et al. 2014) or an a priori box-wing model as in ESA (Flohrer et al. 2015) and CNES (Loyer et al. 2012), or by using an a priori model with a different decomposition of main axes for the empirical accelerations as proposed by Montenbruck et al. (2015a, b). However, all these solutions are based on the estimation of empirical orbit parameters which may absorb some geophysical signals, e.g., the geocenter motion. Therefore, another alternative could be the measurement of orbit perturbations using onboard accelerometers as proposed by Kalarus et al. (2016) for future Galileo spacecraft.

\subsection{Dependency on the observation time}

Using ECOM1 results in a strong correlation between the SLR residuals and the local time of acquisition of the SLR observations. In case of GLONASS-M, the SLR residuals for daytime tracking are at the level of $-30 \mathrm{~mm}$, whereas for nighttime tracking the residuals are at the level of $+20 \mathrm{~mm}$ (Sośnica et al. 2015). The opposite pattern is found for Galileo satellites when using ECOM1 (see Fig. 12, left): The daytime tracking is associated with the positive residuals of about $+100 \mathrm{~mm}$, whereas the nighttime tracking is associated with negative residuals of $-150 \mathrm{~mm}$. This pattern is directly related to orbit modeling deficiencies as it can substantially be reduced by using ECOM2. Figure 12 (right) illustrates that using ECOM2 reduces the nighttime and daytime dependency by a factor of four: The daytime residuals are reduced to about $-10 \mathrm{~mm}$ and the nighttime residuals to $-70 \mathrm{~mm}$.

However, few SLR residuals with large negative values do still exist, especially for the nighttime hours, as shown in Fig. 12 (right). Only the best SLR stations are able to track high-orbiting targets during the daytime, whereas 

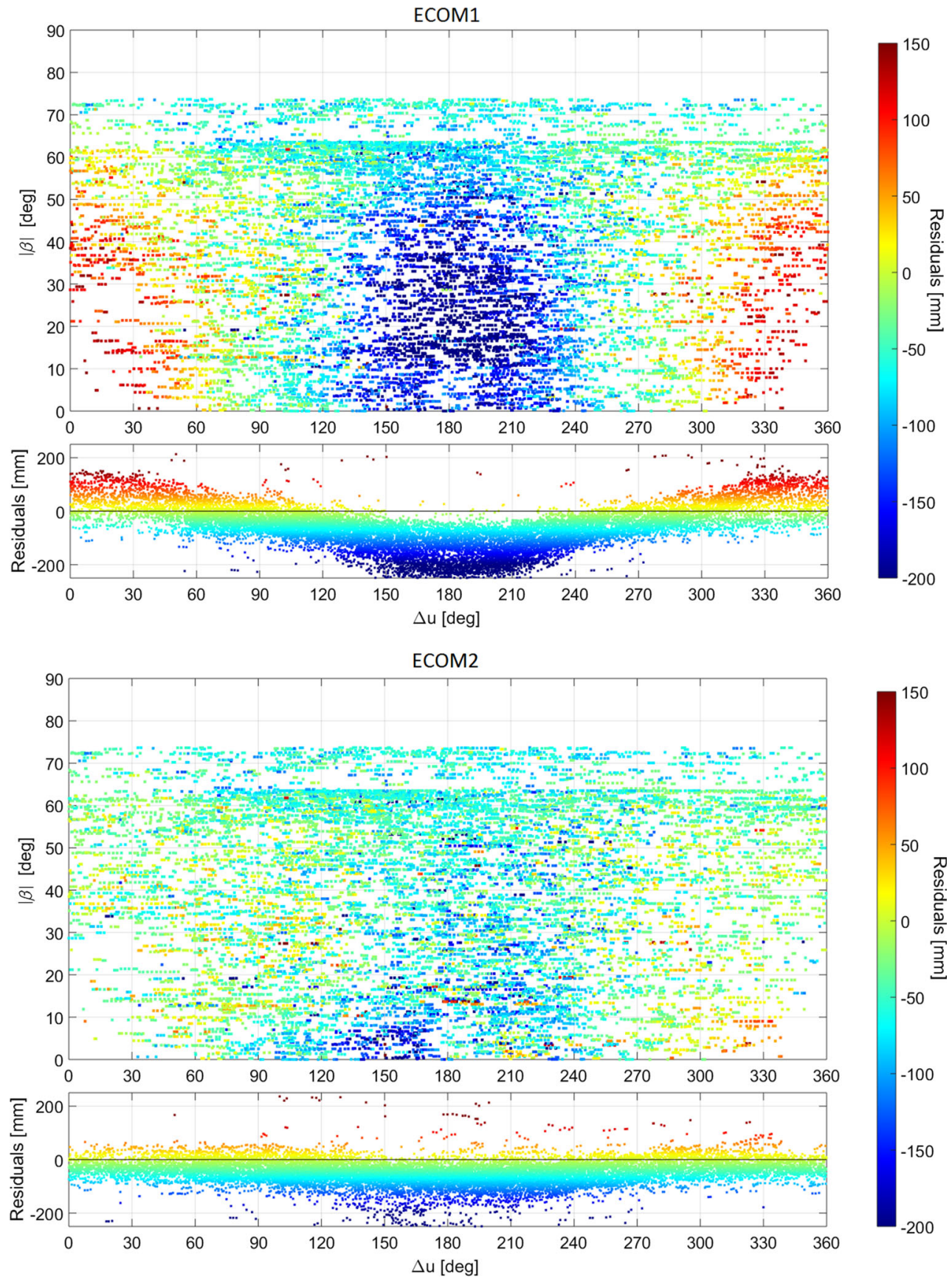

Fig. 11 SLR residuals as a function of the Sun elevation $|\beta|$ and relative Sun-satellite latitude $\Delta u$ angles when using ECOM1 and ECOM2 for Galileo satellites in 2014

almost all SLR stations are capable of tracking GNSS during the nighttime. Therefore, Fig. 13 shows only the SLR residuals from the European C-SPAD stations, which are typically characterized by the highest quality of collected observations (see Sect. 5). Figure 13 (left) shows the SLR residuals to IOV satellites, which suggests that the difference between daytime and nighttime tracking exists even for high-performing stations but is smaller when compared to all SLR stations. The dependency of SLR residuals on the local time of observation acquisition for single-photon stations tracking Galileo satellites in incorrect orbits is smallest out of Galileo satellites (see Fig. 13, right), which confirms a high quality of these orbits. However, a longer time series of observations and a larger number of high-quality SLR observations are needed for a reliable analysis focusing on a quality assessment of individual stations and individual Galileo satellites. 
Fig. 12 Dependency between SLR residuals, local time of an observation acquisition, and the satellite-Sun elongation angle for all Galileo satellites observed in 2014 when using ECOM1 and ECOM2
Fig. 13 Dependency between SLR residuals, local time of an observation acquisition, and the satellite elongation angle for $\mathrm{IOV}$ and FOC in incorrect orbital planes observed in 2014.0-2016.5 by 4 European C-SPAD stations when using ECOM2
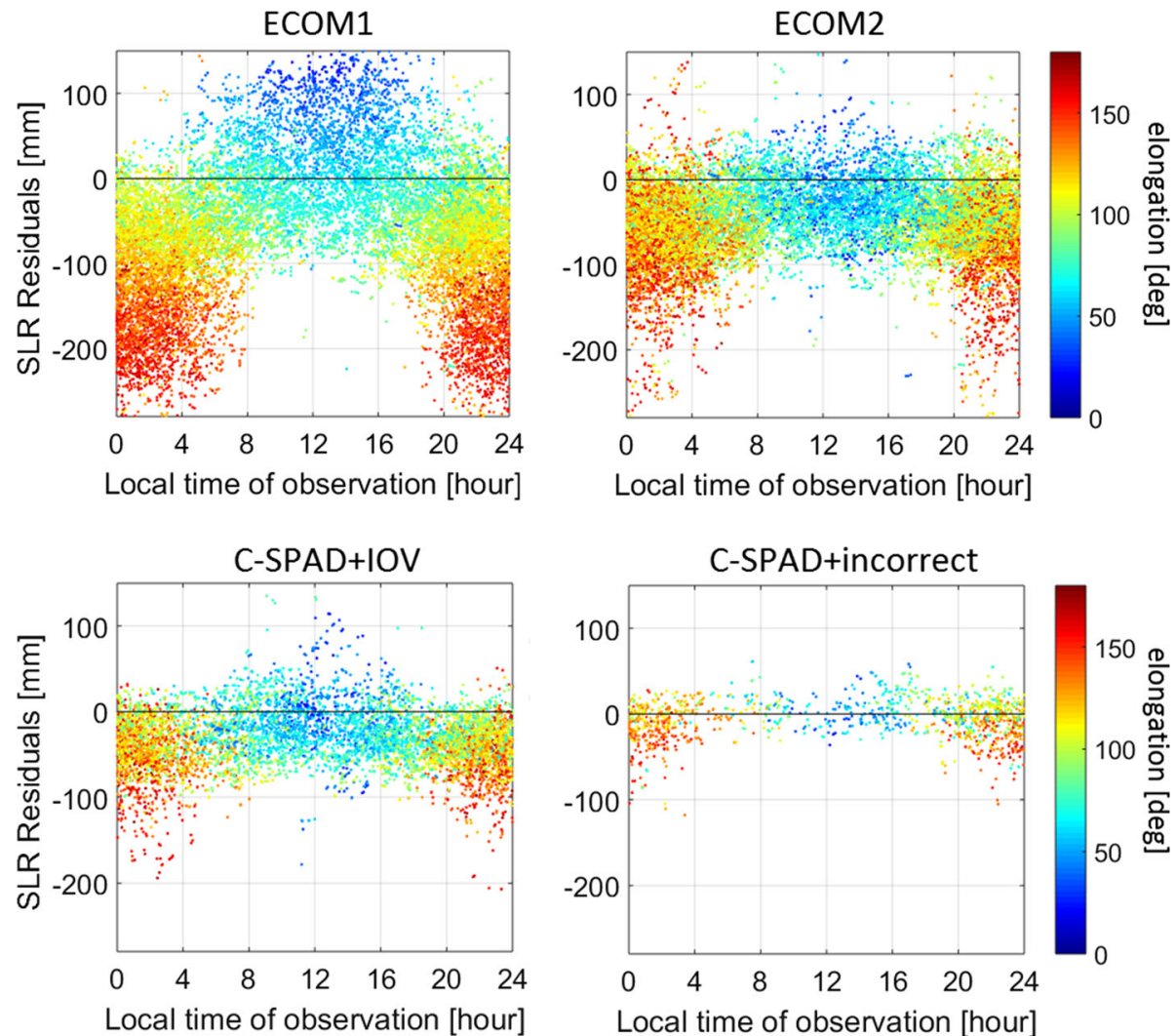

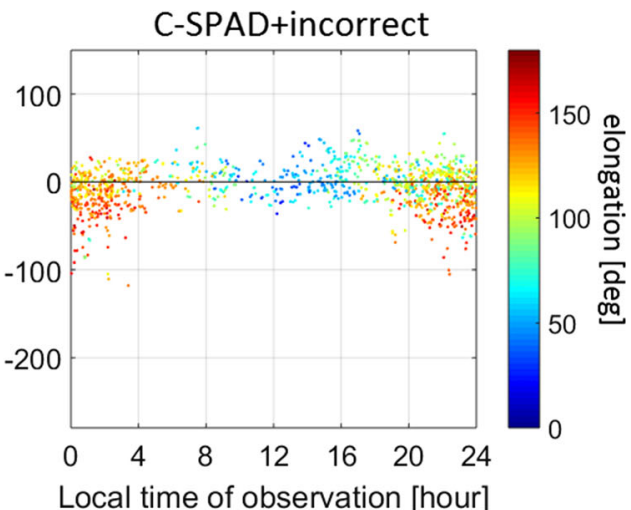

\section{Summary and conclusions}

In this paper, we compared SLR observations with respect to microwave-based Galileo orbits generated by CODE in the framework of the IGS MGEX campaign for the period 2014.0-2016.5. The resulting RMS of SLR residuals is between 29 and $39 \mathrm{~mm}$ for most of the high-performing SLR stations when tracking all Galileo satellites. The mean offset equals to $-43.4,-43.9$, and $-29.5 \mathrm{~mm}$ for selected MCP, PMT, and C-SPAD stations, respectively. Most of the $\mathrm{kHz}$ stations and stations that upgraded from interval timers to event timers were able to significantly reduce their biases. However, the SLR residuals depend not only on the detector type, but also on the generation of Galileo satellites. The smallest offset, amounting to $-6.7 \mathrm{~mm}$, is obtained for the two Galileo satellites in incorrect orbits and when tracked by the European single-photon stations. The mean RMS is then at the level of $23 \mathrm{~mm}$, which is very similar to the quality of SLR observations to GPS-35/36 satellites. The RMS of SLR residuals to the Galileo satellites in incorrect orbital planes equals 22.3, 23.0, and $25.1 \mathrm{~mm}$ for Graz, Herstmonceux, and Zimmerwald, respectively. This confirms the very high quality of Galileo orbits, especially for those satellites which were launched into incorrect orbits.

ECOM1 introduces a very strong dependency between SLR residuals and the satellite's argument of latitude, which results in mean SLR residual variations between $-200 \mathrm{~mm}$ when the satellite is in the opposite direction in the sky w.r.t. the Sun and $+150 \mathrm{~mm}$ when the satellite's argument of latitude is close to zero and the $|\beta|$ angle is low. A similar dependency is also visible for the nighttime and daytime tracking: The daytime SLR residuals are equal to about $+100 \mathrm{~mm}$, whereas the nighttime residuals equal on average to about $-150 \mathrm{~mm}$ when using ECOM1.

The new ECOM2 results in a superior performance with respect to the previously used ECOM1 as it substantially reduces the dependence between SLR residuals and satellites' elongation angles with respect to the Sun's position and the local time of SLR observations. ECOM2 models much better the impact of direct solar radiation pressure acting on a variable surface area of the satellite boxes exposed to the Sun, especially for Galileo satellites whose side surface areas differ remarkably, i.e., $X=1.3$ and $Z=3.0 \mathrm{~m}^{2}$. However, some lesser systematic effects still exist in the SLR residuals especially when $|\beta|<8^{\circ}$ even when using ECOM2. The mean slope of the elongation dependency w.r.t. SLR residuals is $-0.50,-0.43$, and $-0.80 \mathrm{~mm} /{ }^{\circ}$ for IOV, incorrect, and FOC satellites, respectively, when using ECOM2. The smallest slope, and thus also orbits of superior quality in the radial direction, is obtained for the two satellites in incorrect orbital planes, despite the fact that the empirical orbit models were developed for circular and not for highly eccentric satellite orbits with a variable aberration due to changeable angular and linear velocities. 
The correlations between SLR residuals and the beta angles, elongation angles, or the local time of observation acquisition are related to limitations in modeling of the impact of solar radiation pressure and other non-gravitational forces acting upon GNSS satellites. Modeling of Galileo satellite orbits is very challenging, due to a large area-tomass ratio of these satellites, which causes high sensitivity of satellite orbits to non-gravitational orbit perturbations. With ECOM1, the modeling errors were much larger than in case when using ECOM2, due to a limited number of estimated empirical orbit parameters, which turned out to be insufficient to appropriately absorb the impact of solar radiation pressure. Extending the number of estimated parameters in ECOM2, especially adding twice-per-revolution parameters in the $D$ direction, substantially reduces spurious signals observed in SLR residuals. However, SLR residuals still reveal some imperfectnesses of ECOM2; thus, further investigation related to a proper modeling of forces acting upon Galileo satellites is indispensable in order to achieve Galileo orbit models freed from any systematic errors.

In 2015, the GREAT experiment (Galileo gravitational Redshift test with Eccentric sATellites) has been launched with the goal to test the gravitational redshift using the onboard atomic clocks on Galileo satellites in elliptical orbits (Delva et al. 2015). The unintended elliptic orbits induce periodic modulation of the gravitational redshift, while their very good clock stability allows measuring this periodic modulation very accurately. ILRS supports the GREAT experiment by organizing a series of intensive tracking campaigns for Galileo satellites to make significant progress in disentangling the desired redshift signals from systematics due to the clock, orbital modeling, target signatures, and tracking system specific errors.

High quality of orbital ephemerides for the two Galileo satellites in incorrect orbits is thus not only crucial for precise positioning and geodesy, but also constitutes a prerequisite for the validation of general relativity effects using highquality Galileo clocks. All orbit modeling deficiencies in the radial direction affect the estimation of GNSS clock products. Fortunately, SLR observations are good proxies of the quality of the radial component and thus may help evaluate and improve both the orbit quality in the radial direction and the clocks, whose utmost quality will be essential for the confirmation of relativistic effects.

Acknowledgements The ILRS and IGS are acknowledged for providing SLR and GNSS data. The SLR and GNSS stations are acknowledged for a continuous tracking of the geodetic satellites as well as for providing high-quality SLR and GNSS observations. K. Sośnica is supported by the Foundation for Polish Science (FNP). K. Sośnica, G. Bury and M. Drożdżewski are supported by the Polish National Science Centre (NCN), Grant No. UMO-2015/17/B/ST10/03108.
Open Access This article is distributed under the terms of the Creative Commons Attribution 4.0 International License (http://creativecomm ons.org/licenses/by/4.0/), which permits unrestricted use, distribution, and reproduction in any medium, provided you give appropriate credit to the original author(s) and the source, provide a link to the Creative Commons license, and indicate if changes were made.

\section{References}

Appleby G, Rodríguez J (2016) NSGF report. Presented at ILRS ASC meeting Vienna 2016. http://ilrs.gsfc.nasa.gov/docs/2016/ILRS_ ASC_EGU2016_TUW_PRESENTATIONS.pdf

Appleby G, Rodríguez J, Altamimi Z (2016) Assessment of the accuracy of global geodetic satellite laser ranging observations and estimated impact on ITRF scale: estimation of systematic errors in LAGEOS observations 1993-2014. J Geod 90(12):1371-1388. doi:10.1007/s00190-016-0929-2

Arnold D, Meindl M, Beutler G, Dach R, Schaer S, Lutz S, Prange L, Sośnica K, Mervart L, Jaeggi A (2015) CODEs new solar radiation pressure model for GNSS orbit determination. J Geod 89(8):775791. doi:10.1007/s00190-015-0814-4

Beutler G, Brockmann E, Gurtner W, Hugentobler U, Mervart L, Rothacher M (1994) Extended orbit modeling techniques at the CODE processing center of the international GPS service for geodynamics (IGS): theory and initial results. Manuscr Geod 19:367-386

Dach R, Lutz S, Walser P, Fridez P (eds) (2015) Bernese GNSS software version 5.2. user manual. Astronomical Institute, University of Bern, Bern Open Publishing, Bern. doi:10.7892/boris.72297

Dell'Agnello SD et al (2010) Creation of the new industry-standard space test of laser retroreflectors for the GNSS and LAGEOS. Adv Space Res 47(5):822-842. doi:10.1016/j.asr.2010.10.022

Delva P, Hees A, Bertone S, Richard E, Wolf P (2015) Test of the gravitational redshift with stable clocks in eccentric orbits: application to Galileo satellites 5 and 6. Class Quantum Gravity 32(23):232003

Dong X, Han X, Fan C, Song Q (2016) Improvements of Changchun SLR station. In: Proceedings from the 20th international workshop on laser ranging, Potsdam, Germany, October 9-14, 2016

Dow JM, Neilan RE, Rizos C (2009) The international GNSS service in a changing landscape of global navigation satellite systems. J Geod 83(3-4):191-198. doi:10.1007/s00190-008-0300-3

Ebert K, Oesterlin W (2008) Dynamic yaw steering method for spacecraft. European patent specification EP 1526072B1

Flohrer C, Springer T, Otten M, Garcia-Serrano C, Dilssner F, Schoenemann E, Enderle W (2015) Using SLR for GNSS orbit model validation. In: Proceedings from the ILRS technical workshop 2015, Matera, Italy

Fritsche M, Sośnica K, Rodríguez-Solano C, Steigenberge P, Dietrich R, Dach R, Wang K, Hugentobler U, Rothacher M (2014) Homogeneous reprocessing of GPS, GLONASS and SLR observations. J Geod 88(7):625-642. doi:10.1007/s00190-014-0710-3

Guo J, Xu X, Zhao Q, Liu J (2015) Precise orbit determination for quadconstellation satellites at Wuhan University: strategy, result validation, and comparison. J Geod. doi:10.1007/s00190-015-0862-9

Guo F, Li X, Zhang X, Wang J (2017) Assessment of precise orbit and clock products for Galileo, BeiDou, and QZSS from IGS MultiGNSS Experiment (MGEX). GPS Solut 21(1):279-290. doi:10. 1007/s10291-016-0523-3

Hackel S, Steigenberger P, Hugentobler U, Uhlemann M, Montenbruck O (2015) Galileo orbit determination using combined GNSS and SLR observations. GPS Solut 19:15-25. doi:10.1007/ s10291-013-0361-5

ILRS (2014) ILRS study group LARGE: laser ranging to GNSS s/c experiment expanded SLR tracking of GNSS satellites. http://ilrs. 
gsfc.nasa.gov/docs/2014/GNSS_Pilot_Project_v2_20140311. pdf

Kalarus M, Wielgosz A, Liwosz T, Sośnica K, Zieliński J (2016) Possible advantages of equipping the GNSS satellites with on-board accelerometers. In: Proceedings of the IAG commission 4 positioning and applications symposium, Wroclaw, Poland, September 4-7, 2016

Kasho S (2014) Accuracy evaluation of QZS-1 precise ephemerides with satellite laser ranging. In: 19th international workshop on laser ranging, 30 October 2014, Annapolis, Maryland

Kirchner G, Koidl F (2015) Maximizing the output of SLR station Graz: tracking 140 targets. In: Proceedings of the 2015 ILRS technical workshop, October 26-30, 2015, Matera, Italy

Kucharski D, Kirchner G, Otsubo T, Koidl F (2015) A method to calculate zero-signature satellite laser ranging normal points for millimeter geodesy - a case study with Ajisai. Earth Planets Space 67:34. doi:10.1186/s40623-015-0204-4

Loyer S, Perosanz F, Mercier F, Capdeville H, Marty JC (2012) Zero difference GPS ambiguity resolution at CNES-CLS IGS Analysis Center. J Geod 86(11):991-1003. doi:10.1007/ s00190-012-0559-2

Lutz S, Meindl M, Steigenberger P, Beutler G, Sośnica K, Schaer S, Dach R, Arnold D, Thaller D, Jäggi A (2016) Impact of the arc length on GNSS analysis results. J Geod 90(4):365-378. doi:10. 1007/s00190-015-0878-1

Montenbruck O, Rizos C, Weber R, Weber G, Neilan RE, Hugentobler U (2013) Getting a grip on multi-GNSS: the international GNSS service MGEX campaign. GPS World 24(7):44-49

Montenbruck O, Schmid R, Mercier F, Steigenberger P, Noll C, Fatkulin R, Kogure S, Ganeshan AS (2015) GNSS satellite geometry and attitude models. Adv Space Res 56(6):1015-1029. doi:10.1016/j. asr.2015.06.019

Montenbruck O, Steigenberger P, Hugentobler U (2015b) Enhanced solar radiation pressure modeling for Galileo satellites. J Geod 89:283-297. doi:10.1007/s00190-014-0774-0

Otsubo T, Appleby GM (2003) System-dependent center-of-mass correction for spherical geodetic satellites. J Geophys Res Solid Earth 108(B4):2201. doi:10.1029/2002JB002209

Otsubo T, Appleby G, Gibbs P (2001) Glonass laser ranging accuracy with satellite signature effect. SGEO 22(56):509-516. doi:10. 1023/A:1015676419548

Otsubo T, Kunimori H, Noda H, Hanada H, Araki H, Katayama M (2011) Asymmetric dihedral angle offsets for large-size lunar laser ranging retroreflectors. Earth Planet Space 63:e13. doi:10.5047/ eps.2011.11.001

Otsubo T, Sherwood RA, Appleby GM, Nuebert R (2015) Centerof-mass corrections for sub-cm-precision laser-ranging targets: Starlette, Stella and LARES. J Geod 89:303. doi:10.1007/ s00190-014-0776-y

Pavlis EC (1995) Comparison of GPS S/C orbits determined from GPS and SLR tracking data. Adv Space Res 16(12):55-58. doi:10.1016/ 0273-1177(95)98780-R

Pearlman MR, Degnan JJ, Bosworth JM (2002) The international laser ranging service. Adv Space Res 30(2):135-143. doi:10.1016/ S0273-1177(02)00277-6

Petit G, Luzum B (eds) (2011) IERS conventions 2010. IERS technical note 36. Verlag des Bundesamts fr Kartographie und Geodäsie, Frankfurt am Main, 2010

Prange L, Dach R, Lutz S, Schaer S, Jäggi A (2016) The CODE MGEX orbit and clock solution. In: Rizos C, Willis P (eds) IAG 150 years, international association of geodesy symposia. Springer, Cham, pp 767-773. doi:10.1007/1345_2015_161
Prange L, Orliac E, Dach R, Arnold D, Beutler G, Schaer S, Jäggi A (2017) CODE's five-system orbit and clock solution-the challenges of multi-GNSS data analysis. J Geod 91(4):345-360. doi:10.1007/s00190-016-0968-8

Rodriguez-Solano C, Hugentobler U, Steigenberger P, Lutz S (2012) Impact of Earth radiation pressure on GPS position estimates. J Geod 86(5):309-317. doi:10.1007/s00190-011-0517-4

Rodriguez-Solano C, Hugentobler U, Steigenberger P, Blossfeld M, Fritsche M (2014) Reducing the draconitic errors in GNSS geodetic products. J Geod 88:559. doi:10.1007/s00190-014-0704-1

Sośnica K, Thaller D, Dach R, Steigenberger P, Beutler G, Arnold D, Jäggi A (2015) Satellite laser ranging to GPS and GLONASS. J Geod 89(7):725-743. doi:10.1007/s00190-015-0810-8

Springer T, Beutler G, Rothacher M (1999) A new solar radiation pressure model for GPS satellites. GPS Solut 3(2):50-62. doi:10.1007/ PL00012757

Springer T, Otten M, Flohrer C (2012) Spreading the usage of NAPEOS, the ESA tool for satellite geodesy. Geophysical Research Abstracts 14, sRef-ID: EGU2012-7099-2, European Geosciences Union General Assembly

Steigenberger P, Montenbruck O (2017) Galileo status: orbits, clocks, and positioning. GPS Solut 21(2):319-331. doi:10.1007/ s10291-016-0566-5

Steigenberger P, Hugentobler U, Hauschild A, Montenbruck O (2013) Orbit and clock analysis of Compass GEO and IGSO satellites. J Geod 87(6):515-525. doi:10.1007/s00190-013-0625-4

Steigenberger P, Hugentobler U, Loyer S, Perosanz F, Prange L, Dach R, Uhlemann M, Gendt G, Montenbruck O (2015) Galileo orbit and clock quality of the IGS Multi-GNSS Experiment. Adv Space Res 55(1):269-281. doi:10.1016/j.asr.2014.06.030

Steigenberger P, Thölert S, Montenbruck O (2017) Determination of GNSS satellite transmit power and impact on orbit determination. Geophys Res Abstr 19:12871

Thaller D, Dach R, Seitz M, Beutler G, Mareyen M, Richter B (2011) Combination of GNSS and SLR observations using satellite co-locations. J Geod 85(5):257-272. doi:10.1007/ s00190-010-0433-z

Thaller D, Sośnica K, Dach R, Jäggi A, Beutler G, Mareyen M, Richter B (2014) Geocenter co-ordinates from GNSS and combined GNSS-SLR solutions using satellite co-locations. In: Earth on the edge: science for a sustainable planet, international association of geodesy symposia, vol 139, pp 129-134. doi:10.1007/ 978-3-642-37222-3_16

Thaller D, Sośnica K, Steigenberger P, Roggenbuck O, Dach R (2015) Pre-combined GNSS-SLR solutions: what could be the benefit for the ITRF? In: van Dam T (ed) REFAG 2014. International association of geodesy symposia, vol 146. Springer, Cham, pp 85-94. doi:10.1007/1345_2015_215

Uhlemann M, Gendt G, Ramatschi M, Deng Z (2016) GFZ global multiGNSS network and data processing results. In: Rizos C, Willis $P$ (eds) IAG 150 years, international association of geodesy symposia. Springer, Cham, pp 673-679. doi:10.1007/1345_2015_120

Urschl C, Beutler G, Gurtner W, Hugentobler U, Schaer S (2007) Contribution of SLR tracking data to GNSS orbit determination. Adv Space Res 39(10):1515-1523. doi:10.1016/j.asr.2007.01.038

Wilkinson M, Appleby G (2011) In-orbit assessment of laser retroreflector efficiency onboard high orbiting satellites. Adv Space Res 48(3):578. doi:10.1016/j.asr.2011.04.008 